Imperial-TP-AT-2016-01

\title{
Iterating free-field AdS/CFT: higher spin partition function relations
}

\author{
Matteo Beccaria ${ }^{1 a}$ and Arkady A. Tseytlin ${ }^{2 b}$ \\ ${ }^{a}$ Dipartimento di Matematica e Fisica Ennio De Giorgi, \\ Università del Salento \& INFN, Via Arnesano, 73100 Lecce, Italy \\ ${ }^{b}$ The Blackett Laboratory, Imperial College, London SW7 2AZ, U.K.
}

\begin{abstract}
We find a simple relation between a free higher spin partition function on thermal quotient of $\mathrm{AdS}_{d+1}$ and the partition function of the associated $d$-dimensional conformal higher spin field defined on thermal quotient of $\mathrm{AdS}_{d}$. Starting with a conformal higher spin field defined in $\mathrm{AdS}_{d}$ one may also associate to it another conformal field in $d-1$ dimensions, thus "iterating" AdS/CFT. We observe that in the case of $d=4$ this iteration leads to a "trivial" $3 \mathrm{~d}$ higher spin conformal theory with parity-even non-local action: it describes zero total number of dynamical degrees of freedom and the corresponding partition function is equal to 1 .
\end{abstract}

\footnotetext{
${ }^{1}$ matteo.beccaria@le.infn.it

${ }^{2}$ Also at Lebedev Institute, Moscow. tseytlin@imperial.ac.uk
} 


\section{Contents}

1 Introduction 1

2 Some general relations 4

3 Partition functions on $\mathrm{AdS}_{d+1}$ and $\mathrm{AdS}_{d} \quad 6$

$\begin{array}{ll}3.1 \text { Conformal scalar } & 6\end{array}$

$\begin{array}{ll}3.2 & \text { Conformal higher spins }\end{array}$

3.3 Conformal symmetric tensors in $d=4 \quad 8$

4 From 5 to 4 to 3 dimensions 9

4.1 $\mathrm{AdS}_{5} \rightarrow \mathrm{AdS}_{4}$

4.2 Relation between partition functions on $\mathrm{AdS}_{4}$ and $S^{1} \times S^{3} \quad 11$

4.3 Further descent: $\mathrm{AdS}_{4} \rightarrow \mathbb{R} \times S^{2} \quad 12$

$\begin{array}{lll}\text { 4.3.1 Spin } 1 & 13\end{array}$

$\begin{array}{lll}4.3 .2 & \text { Spin } 2 & 15\end{array}$

5 Concluding remarks 16

$\begin{array}{lll}\text { A Reconstructing } \mathcal{Z}_{\mathrm{CF}}^{+}\left(\mathbf{A d S}_{d}\right) \text { from } \mathcal{Z}_{\mathrm{HS}}^{+}\left(\mathrm{AdS}_{d+1}\right) & 17\end{array}$

B $\sigma$-term relation in Eq. (3.4) 19

$\begin{array}{lr}\text { C Higher derivative conformal scalar fields } & 19\end{array}$

$\begin{array}{ll}\text { C.1 Partition function on } S^{1} \times S^{3} & 20\end{array}$

C.2 Partition function on $\mathrm{AdS}_{d} \quad 21$

$\begin{array}{ll}\text { D Fermionic conformal higher spin fields } & 21\end{array}$

E Conformal antisymmetric tensor fields in 4d 22

\section{Introduction}

"Kinematical" AdS/CFT correspondence relates a field $\varphi$ in $\mathrm{AdS}_{d+1}$ (e.g., with standard 2-derivative action with some mass parameter $M^{2}$ or associated dimension $\Delta$ ) to a conformal field $\phi$ at the boundary $\mathcal{M}^{d}=\partial\left(\operatorname{AdS}_{d+1}\right)$ with canonical dimension $\Delta^{-}=d-\Delta$. The value of AdS mass parameter and thus of $\Delta^{-}$determines the number of derivatives in the kinetic term in the action for $\phi$ :

$$
S_{d}=\int d^{d} x \phi \partial^{k} \phi, \quad k=d-2 \Delta^{-}=2 \Delta-d .
$$

For example, a massless totally symmetric higher spin field $\varphi_{s}$ in $\operatorname{AdS}_{d+1}$ is associated to a conformal higher spin field $\phi_{s}$ with the action $S_{d}=\int d^{d} x \phi_{s} P_{s} \partial^{2 s+d-4} \phi_{s}$ where $P_{s}$ is 
traceless transverse projector (see [1] for a review and refs.). From the standard AdS/CFT perspective a massless AdS field $\varphi_{S}$ is a counterpart of a bilinear conserved current $J_{S}$ of a free (e.g., scalar $\Phi$ ) boundary $\mathrm{CFT}_{d}$ while $\phi_{s}$ is associated with a shadow field or a source for $J_{s}$; thus the action for $\phi_{s}$ may be interpreted as an "induced" action found upon integrating out $\Phi$ coupled to $\phi_{s}$ via $J_{s}(\Phi)$.

There are other relations between the two free theories $S_{d+1}(\varphi)$ and $S_{d}(\phi)$ in $d+1$ and $d$ dimensions beyond just kinematic $S O(2, d)$ representation theory correspondence. First, the $\operatorname{AdS}_{d+1}$ action for $\varphi$ evaluated on the solution of the Dirichlet problem $\left.\varphi\right|_{\partial}=\phi$ gives an "induced" action for $\phi$. For even $d$ and, e.g., for a massless field $\varphi$ the $\operatorname{AdS}_{d+1}$ action contains a logarithmically divergent local term which is identified with a local action for $\phi$. For odd $d$ one gets, in general, a non-local action as the power $k$ in the kinetic operator in (1.1) may be half-integer or negative. In addition to this tree-level relation there is also a 1-loop one - between ratio of partition functions for a higher spin (HS) field $\varphi$ in $\mathrm{AdS}_{d+1}$ with $\mathrm{D}$ and $\mathrm{N}$ (or + and - ) boundary conditions and for the dual conformal field (CF) $\phi$ at the boundary:

$$
\left.\frac{Z_{\mathrm{HS}}^{-}}{Z_{\mathrm{HS}}^{+}}\right|_{\mathrm{AdS}_{d+1}}=\left.Z_{\mathrm{CF}}\right|_{\partial\left(\mathrm{AdS}_{d+1}\right)} .
$$

This relation is true, e.g., for global $\mathrm{AdS}_{d+1}$ with boundary $S^{d}$ and also for a thermal quotient of $\mathrm{AdS}_{d+1}$ with boundary $S_{\beta}^{1} \times S^{d-1}$. In the latter case (1.2) translates into a relation between one-particle partition functions $\mathcal{Z}$ as functions of the $q=e^{-\beta}$ variable $^{1}$

$$
\begin{aligned}
& \mathcal{Z}_{\mathrm{HS}}^{-}\left(\operatorname{AdS}_{d+1} ; q\right)-\mathcal{Z}_{\mathrm{HS}}^{+}\left(\operatorname{AdS}_{d+1} ; q\right)=\mathcal{Z}_{\mathrm{CF}}\left(S^{1} \times S^{d-1} ; q\right), \\
& Z=\exp \sum_{n=1}^{\infty} \frac{1}{n} \mathcal{Z}\left(q^{n}\right), \\
& \mathcal{Z}^{-}\left(\operatorname{AdS}_{d+1} ; q\right)=\widetilde{\mathcal{Z}}^{-}\left(\operatorname{AdS}_{d+1} ; q\right)+\sigma_{d+1}(q) \\
& \widetilde{\mathcal{Z}}^{-}\left(\operatorname{AdS}_{d+1} ; q\right) \equiv(-1)^{d} \mathcal{Z}^{+}\left(\operatorname{AdS}_{d+1} ; q^{-1}\right) .
\end{aligned}
$$

Eq. (1.3) may be interpreted in terms of counting of operators in the boundary CFT or as a group-theoretic relation for characters of the conformal group. More generally, (1.2) is expected to be true even for asymptotically AdS space and its generic curved boundary (provided the corresponding $d+1$ and $d$ dimensional theories can be consistently defined) and should thus provide, in particular, an AdS theory based way to compute not only the conformal anomaly a-coefficients [2] but also the c-coefficients [3].

Having identified a conformal field $\phi$ in $\mathbb{R}^{d}$ associated to a field $\varphi$ in $\operatorname{AdS}_{d+1}$ we may attempt to repeat this step one more time. Namely, we may first define this $\phi$ not on $\mathbb{R}^{d}$ (or $S^{d}$ or $S^{1} \times S^{d-1}$ ) but on $\mathrm{AdS}_{d}$ and then associate to it another conformal field $\widehat{\phi}$ in $d-1$

\footnotetext{
${ }^{1}$ Here $\sigma_{d}(q)$ is a finite polynomial in $q+q^{-1}$ that represents contribution of finite number of "zero" modes related to gauge invariance of the conformal (shadow) field $[2,1]$.
} 
dimensions. We will then have the following dimensional $(d+1 \rightarrow d \rightarrow d-1)$ digression $^{2}$

$$
\varphi\left(\mathrm{AdS}_{d+1}\right) \rightarrow \phi\left(\partial \mathrm{AdS}_{d+1} \propto \mathrm{AdS}_{d}\right) \rightarrow \widehat{\phi}\left(\partial \mathrm{AdS}_{d}\right)
$$

If $\varphi$ is a gauge field with 2-derivative action in $\mathrm{AdS}_{d+1}$, then $\phi$ is also single (gauge) conformal field with, in general, higher derivative action. The latter can be represented as a collection of 2nd-derivative fields in $\mathrm{AdS}_{d}$ and hence $\widehat{\phi}$ in $d-1$ dimensions will be given by set of several conformal fields, each being dual to an individual 2nd-derivative field in $d$ dimensions.

Our aim below will be to explore some implications of this "iterated" AdS/CFT correspondence (1.7) at the level of relations between partition functions like (1.2) and (1.3). We shall find that for a generic higher spin field (HS) in $\mathrm{AdS}_{d+1}$ and its dual conformal field (CF) in $d$ dimensions one gets also

$$
\mathcal{Z}_{\mathrm{HS}}^{-}\left(\mathrm{AdS}_{d+1} ; q\right)-\mathcal{Z}_{\mathrm{HS}}^{+}\left(\operatorname{AdS}_{d+1} ; q\right)=\mathcal{Z}_{\mathrm{CF}}^{-}\left(\operatorname{AdS}_{d} ; q\right)+\mathcal{Z}_{\mathrm{CF}}^{+}\left(\operatorname{AdS}_{d} ; q\right)
$$

where $\mathcal{Z}^{-}$may be replaced by $\widetilde{\mathcal{Z}}^{-}$in (1.6) as one finds also that the $\sigma$ terms in (1.5) match, $\sigma_{\mathrm{HS}, d+1}=\sigma_{\mathrm{CF}, d}$. Eq. (1.8) follows from (1.3) and

$$
\mathcal{Z}_{\mathrm{CF}}^{-}\left(\mathrm{AdS}_{d} ; q\right)+\mathcal{Z}_{\mathrm{CF}}^{+}\left(\mathrm{AdS}_{d} ; q\right)=\mathcal{Z}_{\mathrm{CF}}\left(S^{1} \times S^{d-1} ; q\right)
$$

which may be related to the fact that $\mathrm{AdS}_{d}$ is conformal to half of $\mathbb{R} \times S^{d-1}$ so that the respective partition functions are related provided one sums over the two possible boundary conditions at the boundary of $\mathrm{AdS}_{d}$. Applying (1.3) to CF in $\mathrm{AdS}_{d}$ and its counterpart conformal field $\widehat{\mathrm{CF}}$ in $d-1$ dimensions (cf. (1.7)) we get also

$$
\mathcal{Z}_{\mathrm{CF}}^{-}\left(\mathrm{AdS}_{d} ; q\right)-\mathcal{Z}_{\mathrm{CF}}^{+}\left(\mathrm{AdS}_{d} ; q\right)=\mathcal{Z}_{\widehat{\mathrm{CF}}}\left(S^{1} \times S^{d-2} ; q\right)
$$

We shall find that the case of $d=4$ is special: starting with a HS field in $\mathrm{AdS}_{5}$, the resulting $3 \mathrm{~d}$ conformal theory represented by $\widehat{\phi}$ is effectively "topological", having zero number of dynamical d.o.f. and trivial partition function. This may be related to equivalence of \pm modes with non-zero spins in $\mathrm{AdS}_{4}$ [4], implying

$$
\mathcal{Z}_{\mathrm{CF}}^{-}\left(\mathrm{AdS}_{4} ; q\right)=\mathcal{Z}_{\mathrm{CF}}^{+}\left(\mathrm{AdS}_{4} ; q\right) \quad \rightarrow \quad \mathcal{Z}_{\widehat{\mathrm{CF}}}\left(S^{1} \times S^{2} ; q\right)=0
$$

Very loosely, this may be interpreted as a version of the "boundary of boundary $=0$ " relation, or as "(AdS $/ \mathrm{CFT})^{2}=0$ ". $^{3}$

\footnotetext{
${ }^{2}$ For standard global $\mathrm{AdS}_{d+1}$ we have $\partial \mathrm{AdS}_{d+1}=\mathbb{R} \times S^{d-1}$. This space is equivalent to two copies of $\mathrm{AdS}_{d}$ glued along their boundary identified with the equator of $S^{d-1}$. The middle step in (1.7) means that we start with the conformal action on $\partial \mathrm{AdS}_{d+1}$ and then translate it into $\mathrm{AdS}_{d}$ (taking also into account the freedom in choice of boundary conditions, see below).

${ }^{3}$ Let us note that our interpretation and examples will be different from previous discussions of "sequential" AdS/CFT like $\mathrm{AdS}_{4} / \mathrm{CF}_{3} \rightarrow \mathrm{AdS}_{3} / \mathrm{CF}_{2}$ in [5, 6] (for related work discussing $\mathrm{AdS}_{d}$ foliations of $\mathrm{AdS}_{d+1}$ see also [7-11]). In particular, in contrast to [5] the $3 \mathrm{~d}$ conformal higher spin theory that will naturally appear in our context is not of local Chern-Simons type but has parity-even non-local action. Let us also mention for completeness that discussions of dimensional reduction from to $\operatorname{AdS}_{d+1}$ to $\operatorname{AdS}_{d}$ appeared in [12, 13].
} 
We shall start in section 2 with a review of some general definitions and relations. Then in section 3 we shall demonstrate the validity of (3.1) on several examples, in particular, for massless higher spin fields in $\mathrm{AdS}_{d+1}$ related to conformal higher spin fields in $\mathrm{AdS}_{d}$.

In section 4 we shall first analyse the detailed structure of the relation (3.1) on the example of the totally symmetric field in $\mathrm{AdS}_{5}$ with generic mass parameter and mention its possible group-theoretic interpretation and then justify the $\mathcal{Z}^{-}=\mathcal{Z}^{+}$equality in (1.11). We shall then discuss in detail the corresponding $3 \mathrm{~d}$ conformal theory with non-local linearized action describing total of zero degrees of freedom and leading to trivial partition function. We shall use spin 1 Maxwell and spin 2 conformal graviton fields as examples.

Section 5 will contain some concluding remarks. In Appendix A we shall discuss the algebraic structure of the partition functions appearing in (3.1) and then in Appendix B argue for the equality of the corresponding $\sigma$-terms in (1.5). In Appendices C, D and $\mathrm{E}$ the relation (3.1) will be further illustrated on the examples of conformal higher derivative scalars, fermionic conformal higher spin fields and conformal antisymmetric tensor field in $4 \mathrm{~d}$.

\section{Some general relations}

Let us consider a conformally invariant action in $\mathrm{AdS}_{d}$. This space is conformally equivalent to one half of static Einstein universe $S^{1} \times S^{d-1}$, with the boundary of $\mathrm{AdS}_{d}$ being mapped to the equator of $S^{d-1}[4,14,15]$. One can consider the single particle partition function $\mathcal{Z}\left(\mathrm{AdS}_{d} ; q\right)$ on thermal $\mathrm{AdS}_{d}$ where we identify $t \sim t+\beta$. This can be compared with the partition function in Einstein universe $\mathcal{Z}\left(S^{1} \times S^{d-1} ; q\right)$ where $S^{1}$ is the thermal circle with length $\beta$.

The calculation of total partition function $Z\left(\mathrm{AdS}_{d} ; q\right)$ (and thus of $\mathcal{Z}\left(\operatorname{AdS}_{d} ; q\right)$ ) is straightforward assuming that the kinetic operator of a conformal field factorises, i.e. the action in $\mathrm{AdS}_{d}$ can be written a sum of 2nd-derivative terms (as, e.g., in [16]). For example, let us consider

$$
\log Z\left(\mathrm{AdS}_{d}\right)=-\frac{1}{2} \sum_{i=1}^{N} n_{i} \log \operatorname{det} \widehat{\Delta}_{s_{i} \perp}\left(M_{i}^{2}\right), \quad \widehat{\Delta}_{s \perp}\left(M^{2}\right) \equiv\left(-\nabla^{2}-M^{2}\right)_{s \perp}
$$

where $\widehat{\Delta}_{s \perp}$ is defined on transverse traceless symmetric tensors of rank $s^{4}$, and the integers $n_{i}$ are field multiplicities positive for physical fields and negative for ghost fields. For each operator in (2.1) the value of mass term then determines possible ground state energies $\Delta_{d}^{ \pm}$that are solutions of the quadratic equation [17-19]

$$
\Delta_{d}^{ \pm}\left(\Delta_{d}^{ \pm}-d+1\right)-s=-M^{2}, \quad \Delta_{d}^{-}=d-1-\Delta_{d}^{+}, \quad \Delta_{d}^{-} \leq \Delta_{d}^{+},
$$

and are associated with classical solutions of the wave equation $\widehat{\Delta}_{s \perp}\left(M^{2}\right) \varphi_{s \perp}=0$ with two different boundary conditions. Taking the thermal quotient of $\mathrm{AdS}_{d}$, we then get

\footnotetext{
${ }^{4}$ In general, we define $\widehat{\Delta}_{S \perp}\left(M^{2}\right)=\left(-\nabla^{2}+M^{2} \epsilon\right)_{s \perp}$, where $\epsilon=-1$ for $\operatorname{AdS}_{d}$ and $\epsilon=+1$ for $S^{d}$ (here we set the curvature scale to 1$)$.
} 
from (2.1) the following two possibilities for the corresponding single particle partition function $\left(q=e^{-\beta}\right)$

$$
\mathcal{Z}^{+}\left(\operatorname{AdS}_{d} ; q\right)=\sum_{i=1}^{N} n_{i} g_{s_{i}}^{(d)} \frac{q^{\Delta_{d, i}^{+}}}{(1-q)^{d-1}}, \quad \widetilde{\mathcal{Z}}^{-}\left(\mathrm{AdS}_{d} ; q\right)=\sum_{i=1}^{N} n_{i} g_{s_{i}}^{(d)} \frac{q^{\Delta_{d, i}^{-}}}{(1-q)^{d-1}} .
$$

In $(2.3) g_{s}^{(d)}$ is the multiplicity that counts the number of off-shell degrees of freedom ${ }^{5}$

$$
g_{s}^{(d)}=(2 s+d-3) \frac{(s+d-4) !}{(d-3) ! s !} .
$$

Using that $\Delta^{-}=d-1-\Delta^{+}$we find

$$
\widetilde{\mathcal{Z}}^{-}\left(\mathrm{AdS}_{d} ; q\right)=(-1)^{d-1} \mathcal{Z}^{+}\left(\operatorname{AdS}_{d} ; q^{-1}\right) .
$$

In the presence of gauge invariance the proper $\mathcal{Z}^{-}\left(\mathrm{AdS}_{d} ; q\right)$ partition function differs from $\widetilde{\mathcal{Z}}^{-}\left(\mathrm{AdS}_{d} ; q\right)$

$$
\mathcal{Z}_{\mathrm{HS}}^{-}\left(\operatorname{AdS}_{d} ; q\right)=\widetilde{\mathcal{Z}}_{\mathrm{HS}}^{-}\left(\operatorname{AdS}_{d} ; q\right)+\sigma_{d}(q)
$$

where $\sigma(q)$ is a finite polynomial in $q+q^{-1}$ related to missing gauge transformations discussed in [1].

The calculation of $\mathcal{Z}\left(S^{1} \times S^{d-1} ; q\right)$ on the Einstein universe background is a priori unrelated to the one on $\mathrm{AdS}_{d}$. If the action on generic $\mathcal{M}^{d}$ is known, one may just specialize it to $S^{1} \times S^{d-1}$, factorize the kinetic operator and use the methods discussed in [1]. ${ }^{6}$ Alternatively, one can make use of the conformal map to flat space $\mathbb{R}^{d}$ ("radial quantization") and use flat space operator counting techniques.

At the same time, $\mathcal{Z}\left(S^{1} \times S^{d-1} ; q\right)$ can also be computed starting with the dual theory in $\mathrm{AdS}_{d+1}$ (cf. (1.3)). If $S^{1} \times S^{d-1}$ is interpreted as the boundary of $\mathrm{AdS}_{d+1}$ the corresponding conformally invariant action on $S^{1} \times S^{d-1}$ can be interpreted as "induced" from an action of a dual field in the bulk (see, e.g., $[2,1]$ and refs. there). Let us call a generic tensor bulk field a "higher spin" (HS) one; this name will include the cases of a massive or partially massless or exactly massless higher spin fields in $\mathrm{AdS}_{d+1}$. The dual conformal field at the boundary will be denoted as CF. Then [1]

$$
\mathcal{Z}_{\mathrm{HS}}^{-}\left(\operatorname{AdS}_{d+1} ; q\right)-\mathcal{Z}_{\mathrm{HS}}^{+}\left(\operatorname{AdS}_{d+1} ; q\right)=\mathcal{Z}_{\mathrm{CF}}\left(S^{1} \times S^{d-1} ; q\right) .
$$

In the case of massless higher spin (MHS) field in $\mathrm{AdS}_{d+1}$ having maximal gauge invariance the associated conformal field at the boundary is conformal higher spin (CHS) one and $\sigma_{d+1}$ in (2.6) is non-trivial [1]. ${ }^{7}$

\footnotetext{
${ }^{5}$ Special cases are $g_{s}^{(4)}=2 s+1, g_{s}^{(6)}=\frac{1}{6}(s+1)(s+2)(2 s+3)$.

${ }^{6}$ If one knows the set of masses $M^{2}$ in (2.1) for an action on $\mathrm{AdS}_{d}$, this is not enough to compute the partition function for the same theory on $S^{1} \times S^{d-1}$. The reason is that $M^{2}$ values come from the specialization to $\mathrm{AdS}_{d}$ of the action on a generic curved background $\mathcal{M}^{d}$ where certain combinations of curvature tensor terms lead to mass terms. Specification of this action to $S^{1} \times S^{d-1}$ will then lead to different kinetic term structures, i.e. to different mass terms in the corresponding 2 nd-order operators.

${ }^{7}$ In addition to the quantum "one-loop" relation (2.7) the quadratic actions for HS and CF have also classical relation: evaluating the action of $\mathrm{HS}$ field in $\mathrm{AdS}_{d+1}$ on the solution with boundary data being equal to CF field one gets the action of the CF field as an "induced" one. In even $d$ case the local CF action is the coefficient of the leading logarithmic IR divergence while in odd $d$ case it is finite but non-local.
} 


\section{Partition functions on $\mathrm{AdS}_{d+1}$ and $\mathrm{AdS}_{d}$}

Let us now propose and check on several examples a general relation between partition functions of higher spin field in $\mathrm{AdS}_{d+1}$ and associated conformal field originally "induced" on $\partial \mathrm{AdS}_{d+1}=\mathbb{R} \times S^{d-1}$ or $S^{d}$ but that can then be also defined on $\mathrm{AdS}_{d}$. This relation is (1.8) that we rewrite here for the reader's convenience

$$
\mathcal{Z}_{\mathrm{HS}}^{-}\left(\operatorname{AdS}_{d+1} ; q\right)-\mathcal{Z}_{\mathrm{HS}}^{+}\left(\operatorname{AdS}_{d+1} ; q\right)=\mathcal{Z}_{\mathrm{CF}}^{-}\left(\operatorname{AdS}_{d} ; q\right)+\mathcal{Z}_{\mathrm{CF}}^{+}\left(\operatorname{AdS}_{d} ; q\right)
$$

Heuristically, the relation (3.1) may be motivated as follows. $\mathrm{AdS}_{d}$ is conformal to half of the Einstein universe $S^{1} \times S^{d-1}$ with two possible choices of the boundary conditions at the equator; thus defining the partition function on $S^{1} \times S^{d-1}$ in terms of $\mathrm{AdS}_{d}$ one we may need to sum over the two boundary condition choices,

$$
\mathcal{Z}_{\mathrm{CF}}^{-}\left(\mathrm{AdS}_{d} ; q\right)+\mathcal{Z}_{\mathrm{CF}}^{+}\left(\mathrm{AdS}_{d} ; q\right)=\mathcal{Z}_{\mathrm{CF}}\left(S^{1} \times S^{d-1} ; q\right) .
$$

Combining this with (2.7) then gives (3.5).

Note that starting with a CF field in $\mathrm{AdS}_{d}$ we may also associate to it another conformal field $\widehat{\mathrm{CF}}$ at the $d-1$ boundary and then the analog of (2.7) will read

$$
\mathcal{Z}_{\mathrm{CF}}^{-}\left(\mathrm{AdS}_{d} ; q\right)-\mathcal{Z}_{\mathrm{CF}}^{+}\left(\mathrm{AdS}_{d} ; q\right)=\mathcal{Z}_{\widehat{\mathrm{CF}}}\left(S^{1} \times S^{d-2} ; q\right) .
$$

Furthermore, the $\sigma(q)$ terms in $(2.6)$ for $\mathcal{Z}_{\mathrm{HS}}^{-}\left(\mathrm{AdS}_{d+1} ; q\right)$ and $\mathcal{Z}_{\mathrm{CF}}^{-}\left(\mathrm{AdS}_{d} ; q\right)$ appear to match (see Appendix B)

$$
\sigma_{\mathrm{HS}, d+1}(q)=\sigma_{\mathrm{CF}, d}(q)
$$

so that (3.1) may be written also as

$$
\widetilde{\mathcal{Z}}_{\mathrm{HS}}^{-}\left(\operatorname{AdS}_{d+1} ; q\right)-\mathcal{Z}_{\mathrm{HS}}^{+}\left(\operatorname{AdS}_{d+1} ; q\right)=\widetilde{\mathcal{Z}}_{\mathrm{CF}}^{-}\left(\operatorname{AdS}_{d} ; q\right)+\mathcal{Z}_{\mathrm{CF}}^{+}\left(\operatorname{AdS}_{d} ; q\right) \text {. }
$$

Using (2.5) this can be put also in the following more symmetric form

$$
-\mathcal{Z}_{\mathrm{HS}}^{+}\left(\operatorname{AdS}_{d+1} ; q\right)+(-1)^{d} \mathcal{Z}_{\mathrm{HS}}^{+}\left(\operatorname{AdS}_{d+1} ; q^{-1}\right)=\mathcal{Z}_{\mathrm{CF}}^{+}\left(\operatorname{AdS}_{d} ; q\right)-(-1)^{d} \mathcal{Z}_{\mathrm{CF}}^{+}\left(\operatorname{AdS}_{d} ; q^{-1}\right)
$$

Below we will demonstrate the validity of (3.1),(3.5) and (3.2) on several examples of conformal fields (for some consequences of (3.5) see also Appendix A).

\subsection{Conformal scalar}

Let us start with the case of a particular scalar field in $\mathrm{AdS}_{d+1}$ with the mass term $M^{2}=$ $\frac{1}{4} d^{2}+1$, i.e. with $\Delta_{d+1}^{ \pm}=\frac{1}{2}(d \pm 2)$ (cf. (2.2)). The corresponding partition (2.3) function is

$$
\mathcal{Z}_{0}^{ \pm}\left(\operatorname{AdS}_{d+1} ; q\right)=\frac{q^{\frac{1}{2}(d \pm 2)}}{(1-q)^{d}}
$$

This scalar in $\mathrm{AdS}_{d+1}$ "induces" a spin 0 field $\varphi$ at the boundary with canonical dimension $=\Delta_{d+1}^{-}=\frac{1}{2}(d-2)$, i.e. which thus represents a conformally coupled scalar. The corresponding kinetic operator in a curved $d$-dimensional space specified to the case of the unit-scale $\mathrm{AdS}_{d}$ (with $R=-d(d-1)$ is

$$
-\nabla^{2}+\frac{d-2}{4(d-1)} R=-\nabla^{2}-\frac{1}{4} d(d-2) .
$$


Thus defining $\varphi$ on $\mathrm{AdS}_{d}$ we find that the mass term (cf. (2.1)) is $M^{2}=\frac{1}{4} d(d-2)$ and thus from (2.2)

$$
\Delta_{d}^{+}=\frac{d}{2}, \quad \Delta_{d}^{-}=\frac{d-2}{2} .
$$

From (2.3) the partition functions corresponding to this conformal scalar (cs) are then

$$
\mathcal{Z}_{\mathrm{cs}}^{+}\left(\operatorname{AdS}_{d} ; q\right)=\frac{q^{d / 2}}{(1-q)^{d-1}}, \quad \quad \mathcal{Z}_{\mathrm{cs}}^{-}\left(\operatorname{AdS}_{d} ; q\right)=(-1)^{d+1} \mathcal{Z}_{\mathrm{cs}}^{+}\left(\operatorname{AdS}_{d} ; q^{-1}\right)
$$

Comparing to (3.7) one can then check that

$$
\mathcal{Z}_{0}^{-}\left(\mathrm{AdS}_{d+1} ; q\right)-\mathcal{Z}_{0}^{+}\left(\mathrm{AdS}_{d+1} ; q\right)=\mathcal{Z}_{\mathrm{cs}}^{-}\left(\operatorname{AdS}_{d} ; q\right)+\mathcal{Z}_{\mathrm{cs}}^{+}\left(\mathrm{AdS}_{d} ; q\right),
$$

which is a particular spin 0 case of (3.1).

To demonstrate (3.2) we recall that the partition function on $S^{1} \times S^{d-1}$ can be found, e.g., by the operator counting method. For a scalar $\varphi$ with canonical dimension $\frac{1}{2}(d-2)$ and equations of motion $\partial^{2} \varphi=0$ that gives

$$
\mathcal{Z}_{\mathrm{cS}}\left(S^{1} \times S^{d-1} ; q\right)=\frac{q^{\frac{1}{2}(d-2)}-q^{\frac{1}{2}(d+2)}}{(1-q)^{d}} .
$$

Then one can check that (2.7) is satisfied (there is no gauge invariance so $\sigma(q)=0$ in (2.6)). As a result, we verify a special case of (3.2)

$$
\mathcal{Z}_{\mathrm{cs}}\left(S^{1} \times S^{d-1} ; q\right)=\mathcal{Z}_{\mathrm{cs}}^{-}\left(\mathrm{AdS}_{d} ; q\right)+\mathcal{Z}_{\mathrm{cs}}^{+}\left(\operatorname{AdS}_{d} ; q\right) .
$$

As already mentioned above, this relation means that one needs to sum over both \pm scalar modes in $\mathrm{AdS}_{d}$ in order to match the conformal scalar partition function on $S^{1} \times S^{d-1}$ space which is conformally equivalent to a double copy of $\mathrm{AdS}_{d}$.

The above discussion can be extended to higher derivative GJMS conformal scalars with higher-derivative kinetic operators, see Appendix $C$, again verifying the general relations (3.1)-(3.5).

\subsection{Conformal higher spins}

Let us now consider a totally symmetric spin $s$ CHS field in $d$ dimensions. The $\mathrm{CHS}$ theory defined on $A d S_{d}$ has the following partition function [20, 21]

$$
\begin{aligned}
\mathrm{Z}_{\mathrm{CHS}, \mathrm{s}}\left(\mathrm{AdS}_{d}\right) & =\prod_{k=0}^{s-1}\left[\frac{\operatorname{det} \widehat{\Delta}_{k \perp}\left(M_{k, s}^{2}\right)}{\operatorname{det} \widehat{\Delta}_{s \perp}\left(M_{s, k}^{2}\right)}\right]^{1 / 2} \prod_{k^{\prime}=-\frac{1}{2}(d-4)}^{-1}\left[\frac{1}{\operatorname{det} \widehat{\Delta}_{s \perp}\left(M_{s, k^{\prime}}^{2}\right)}\right]^{1 / 2}, \\
M_{n, k}^{2} & \equiv n-(k-1)(k+d-2) .
\end{aligned}
$$

For each determinant here (cf. (2.1)) we may then compute the corresponding contribution to the one-particle partition function using the general relations (2.2),(2.3). As a result, we get

$$
\mathcal{Z}_{\mathrm{CHS}, s}^{+}\left(\mathrm{AdS}_{d} ; q\right)=\frac{1}{(1-q)^{d-1}}\left\{\sum_{k=0}^{s-1}\left[g_{s}^{(d)} q^{d+k-2}-g_{k}^{(d)} q^{d+s-2}\right]+\sum_{k^{\prime}=-\frac{1}{2}(d-4)}^{-1} g_{s}^{(d)} q^{d+k^{\prime}-2}\right\},
$$




$$
\widetilde{\mathcal{Z}}_{\mathrm{CHS}, s}^{-}\left(\operatorname{AdS}_{d} ; q\right)=(-1)^{d-1} \mathcal{Z}_{\mathrm{CHS}, s}^{+}\left(\operatorname{AdS}_{d} ; q^{-1}\right) .
$$

Doing the sum, we find

$$
\begin{aligned}
\mathcal{Z}_{\mathrm{CHS}, s}^{+}\left(\mathrm{AdS}_{d} ; q\right)= & \frac{\Gamma(d+s-3)}{\Gamma(d-1) \Gamma(s+1)} \frac{q^{\frac{d}{2}-2}}{(1-q)^{d}}\left[(d-2)(d+2 s-3) q^{2}\right. \\
& \left.-(d+s-3)(d+2 s-2) q^{\frac{d}{2}+s}+s(d+2 s-4) q^{\frac{d}{2}+s+1}\right] .
\end{aligned}
$$

In the special case of the $s=2$ CHS field or, equivalently, of Weyl gravity on thermal quotient of $\mathrm{AdS}_{4}$ and $\mathrm{AdS}_{6}$ this partition function was independently computed also in $[22,23]$

$$
\mathcal{Z}_{\mathrm{CHS}, 2}^{+}\left(\mathrm{AdS}_{4} ; q\right)=\frac{q^{2}\left(5+5 q-4 q^{2}\right)}{(1-q)^{3}}, \quad \mathcal{Z}_{\mathrm{CHS}, 2}^{+}\left(\mathrm{AdS}_{6} ; q\right)=\frac{2 q^{3}\left(7+7 q+7 q^{2}-3 q^{3}\right)}{(1-q)^{5}} .
$$

The CHS field in $d$ dimensions is naturally associated to the massless higher spin (MHS) field in $\mathrm{AdS}_{d+1}$ with $\Delta_{d+1}^{+}=d+s-2$. It has the one-particle partition function [24-26]

$$
\begin{aligned}
& \mathcal{Z}_{\mathrm{MHS}, s}^{+}\left(\operatorname{AdS}_{d+1} ; q\right)=\frac{g_{s}^{(d+1)} q^{d+s-2}-g_{s-1}^{(d+1)} q^{s+d-1}}{(1-q)^{d}}, \\
& \widetilde{\mathcal{Z}}_{\mathrm{MHS}, s}^{-}\left(\operatorname{AdS}_{d+1} ; q\right)=(-1)^{d} \mathcal{Z}_{\mathrm{MHS}, s}^{+}\left(\operatorname{AdS}_{d+1} ; q^{-1}\right)
\end{aligned}
$$

where $g_{s}^{(d)}$ is given by (2.4). One can then check that

$$
\widetilde{\mathcal{Z}}_{\mathrm{MHS}, s}^{-}\left(\operatorname{AdS}_{d+1} ; q\right)-\mathcal{Z}_{\mathrm{MHS}, s}^{+}\left(\operatorname{AdS}_{d+1} ; q\right)=\widetilde{\mathcal{Z}}_{\mathrm{CHS}, s}^{-}\left(\operatorname{AdS}_{d} ; q\right)+\mathcal{Z}_{\mathrm{CHS}, s}^{+}\left(\operatorname{AdS}_{d} ; q\right),
$$

which is another special case of (3.5). One can also verify the validity of (3.1) or, equivalently, (3.4) (see Appendix B).

\subsection{Conformal symmetric tensors in $d=4$}

Next, let us discuss the conformal symmetric rank $s$ tensor field (CST) in $d=4$ considered in $[27,28]$. This is a non-unitary theory that may be viewed as a maximal depth $r=s$ representative of the family of FT-type [29] conformal higher spin fields with rank $s-r$ tensor gauge invariance [30-32]. The CHS theory is the minimal depth case (i.e. case of maximal gauge invariance) when $r=1$. The CST field has 2nd-derivative Lagrangian with scalar gauge invariance and corresponds to a "short" representation of $S O(2,4)$ given by

$$
\mathrm{CST}_{s}=\left(1 ; \frac{s}{2}, \frac{s}{2}\right)-(1-s ; 0,0) \text {. }
$$

The partition function for a CST field defined on $\mathrm{AdS}_{4}$ is found to be [28] (cf. (2.1))

$$
\mathrm{Z}_{\mathrm{CST}, s}\left(\mathrm{AdS}_{4}\right)=\prod_{k=1}^{s}\left[\frac{\operatorname{det} \widehat{\Delta}_{0}\left(2-k-k^{2}\right)}{\operatorname{det} \widehat{\Delta}_{k \perp}(2+k)}\right]^{1 / 2} .
$$

Using (2.2),(2.3) we then find for the one-particle partition function on thermal $\mathrm{AdS}_{4}$

$$
\mathcal{Z}_{\mathrm{CST}, s}^{+}\left(\mathrm{AdS}_{4} ; q\right)=-\widetilde{\mathcal{Z}}_{\mathrm{CST}, s}^{-}\left(\mathrm{AdS}_{4} ; q^{-1}\right)=\frac{1}{(1-q)^{3}} \sum_{k=1}^{s}\left[(2 k+1) q^{2}-q^{k+2}\right]
$$




$$
=\frac{q^{2}\left[s(s+2)-(s+1)^{2} q+q^{s+1}\right]}{(1-q)^{4}} .
$$

This 4d CST field corresponds to the maximal-depth partially massless (PM) totally symmetric spin $s$ field in $\mathrm{AdS}_{5}$ associated with the following combination of $S O(2,4)$ representations $[31]^{8}$

$$
\mathrm{PM}_{s}^{(s)}=\left(3 ; \frac{s}{2}, \frac{s}{2}\right)-(3+s ; 0,0),
$$

for which (3.22) is a "shadow" counterpart. Then from (2.3) we get

$$
\mathcal{Z}_{\mathrm{PM}_{s}^{(s)}}^{+}\left(\mathrm{AdS}_{5} ; q\right)=\widetilde{\mathcal{Z}}_{\mathrm{PM}_{s}^{(s)}}^{-}\left(\operatorname{AdS}_{5} ; q^{-1}\right)=\frac{(s+1)^{2} q^{3}-q^{s+3}}{(1-q)^{4}} .
$$

Comparing (3.24) and (3.26) we conclude that

$$
\widetilde{\mathcal{Z}}_{\mathrm{PM}_{s}^{(s)}}^{-}\left(\mathrm{AdS}_{5} ; q\right)-\mathcal{Z}_{\mathrm{PM}_{s}^{(s)}}^{+}\left(\mathrm{AdS}_{5} ; q\right)=\widetilde{\mathcal{Z}}_{\mathrm{CST}, \mathrm{s}}^{-}\left(\mathrm{AdS}_{4} ; q\right)+\mathcal{Z}_{\mathrm{CST}, s}^{+}\left(\mathrm{AdS}_{4} ; q\right)
$$

in agreement with (3.5). As in CHS case, one can also verify the validity of (3.1) also in the CST case. We shall further discuss the properties of 4d CST field partition functions in the next section.

\section{$4 \quad$ From 5 to 4 to 3 dimensions}

In this section we shall consider a special case of $d=4$ where some relation simplify. We shall discuss further descent to 3 dimensions thus getting a "triple" of related fields: $\mathrm{HS}$ in $5, \mathrm{CF}$ in 4 , and $\widehat{\mathrm{CF}}$ in 3 dimensions. In particular, starting with a massless higher spin field in $\mathrm{AdS}_{5}$ one gets a conformal higher spin in $4 \mathrm{~d}$ and then defininig it on $\mathrm{AdS}_{4}$ can further associate to it another conformal higher spin field in $3 \mathrm{~d}$. The latter turns out to have a non-local action describing zero number of dynamical degrees of freedom, i.e. giving trivial partition function.

\section{1 $\mathrm{AdS}_{5} \rightarrow \mathrm{AdS}_{4}$}

Let us first consider the $d=4$ version of the relation (3.1) between partition functions of some higher spin field in $\mathrm{AdS}_{5}$ and the corresponding $4 \mathrm{~d}$ conformal field defined on $\mathrm{AdS}_{4}$. Starting with a totally symmetric spin $s$ HS field in $\mathrm{AdS}_{5}$ corresponding to $S O(2,4)$ representation $\left(\Delta_{5} ; \frac{s}{2}, \frac{s}{2}\right)$ we may associate to it (in general, higher-derivative) conformal field in $4 \mathrm{~d}$ that may also be represented (when defined on $\mathbb{R}^{4}$ or $\mathrm{AdS}_{4}$ ) as a collection of 2nd-derivative fields with particular values of masses. Our proposal for such general relation is ${ }^{9}$

$$
\left(\Delta_{5} ; \frac{s}{2}, \frac{s}{2}\right)_{\mathrm{AdS}_{5}} \longrightarrow Z_{\mathrm{CF}, s}\left(\mathrm{AdS}_{4}\right)=\prod_{s^{\prime}=0}^{s} \prod_{k=0}^{\Delta_{5}-3}\left[\operatorname{det} \widehat{\Delta}_{s^{\prime} \perp}\left(M_{s^{\prime}, k}^{2}\right)\right]^{-1 / 2},
$$

\footnotetext{
${ }^{8}$ The subscript $r$ in $\mathrm{PM}_{s}^{(r)}$ denotes the depth. Here, we consider only the maximal case $r=s$.

${ }^{9} \mathrm{~A}$ similar "correspondence rule" in 6d was discussed in Appendix A of [33].
} 
where $M_{s^{\prime}, k}^{2}=s^{\prime}-2-k(k+1)$ as in (3.15). Special HS fields with gauge invariance will require combinations of the above building blocks to take into account ghost field contributions. One can check that (4.1) is consistent with all special conformal fields in $4 \mathrm{~d}$ that we have analysed directly: CHS, CST and also GJMS scalar fields (see Appendix C).

Given (4.1) one can then demonstrate the validity of the relation (3.1) (equivalent to (3.5) in the absence of gauge invariance) between the partition functions in $\mathrm{AdS}_{5}$ and $\mathrm{AdS}_{4}$. For each factor in the r.h.s. of (4.1) we find from (2.2) that $\Delta_{4, k}^{+}=k+2$ and thus applying (2.3) to both $5 \mathrm{~d}$ and $4 \mathrm{~d}$ cases we get

$$
\begin{aligned}
& \mathcal{Z}_{\left(\Delta_{5} ; \frac{s}{2}, \frac{s}{2}\right)}^{+}\left(\operatorname{AdS}_{5} ; q\right)=(s+1)^{2} \frac{q^{\Delta_{5}}}{(1-q)^{4}} \\
& \mathcal{Z}_{\mathrm{CF}}^{+}\left(\mathrm{AdS}_{4} ; q\right)=\sum_{s^{\prime}=0}^{s} \sum_{k=0}^{\Delta_{5}-3}\left(2 s^{\prime}+1\right) \frac{q^{k+2}}{(1-q)^{3}}=(s+1)^{2} \frac{q^{2}-q^{\Delta_{5}}}{(1-q)^{4}} .
\end{aligned}
$$

Then using the expression (2.5) for $\widetilde{\mathcal{Z}}^{-}$we indeed verify (3.1), i.e.

$$
\widetilde{\mathcal{Z}}_{\left(\Delta_{5} ; \frac{s}{2}, \frac{s}{2}\right)}^{-}\left(\mathrm{AdS}_{5} ; q\right)-\mathcal{Z}_{\left(\Delta_{5} ; \frac{s}{2}, \frac{s}{2}\right)}^{+}\left(\mathrm{AdS}_{5} ; q\right)=\widetilde{\mathcal{Z}}_{\mathrm{CF}}^{-}\left(\mathrm{AdS}_{4} ; q\right)+\mathcal{Z}_{\mathrm{CF}}^{+}\left(\mathrm{AdS}_{4} ; q\right)
$$

To provide additional support for the correspondence rule (4.1) let us consider the CF partition function defined on $S^{4}$ instead of $\mathrm{AdS}_{4}$ which may be viewed as a boundary of global $\mathrm{AdS}_{5}$. In that case we should get an analog of (2.7), i.e. the relation (1.2)

$$
\log Z_{\mathrm{HS}}^{-}\left(\mathrm{AdS}_{5}\right)-\log \mathrm{Z}_{\mathrm{HS}}^{+}\left(\mathrm{AdS}_{5}\right)=\log \mathrm{Z}_{\mathrm{CF}}\left(S^{4}\right) .
$$

One may check this relation by comparing the coefficient of the IR divergent term on the 1.h.s. to the coefficient of the UV divergent term on the r.h.s., i.e. to the $4 \mathrm{~d}$ conformal anomaly a-coefficient $[2,1]$. According to Eq.(3.3) of [1], we get for the coefficient in the 1.h.s.

$$
\mathrm{a}\left(\Delta_{5} ; \frac{s}{2}, \frac{s}{2}\right)=-\frac{1}{720}\left(\Delta_{5}-2\right)^{3}(s+1)^{2}\left[3\left(\Delta_{5}-2\right)^{2}-5 s^{2}-10 s-5\right] .
$$

On the other hand, each $\operatorname{det}\left(-\nabla_{s}^{2}+M^{2}\right)_{s \perp}$ in the product in (4.1) defined on $S^{4}$ gives the contribution (see Eq.(3.37) of [20])

$$
\mathrm{a}_{s \perp}\left(M^{2}\right)=\frac{1}{720}(2 s+1)\left[30 s^{3}+85 s^{2}+10 s-58-30\left(s^{2}-2\right) M^{2}-15 M^{4}\right] .
$$

Then for the particular combination of the operators in (4.1) we get indeed

$$
\mathrm{a}\left(\Delta_{5} ; \frac{s}{2}, \frac{s}{2}\right)=\sum_{k=0}^{\Delta_{5}-3} \sum_{s^{\prime}=0}^{s} \mathrm{a}_{s^{\prime} \perp}\left(M_{s^{\prime}, k}^{2}\right)
$$

Let us note that the relation (4.4), implied by the correspondence rule (4.1), should have a group theoretic interpretation. To see an indication of this, let us consider the "nonblind" characters $\chi_{4}$ and $\chi_{3}$ of massive representations of $S O(4,2)$ and $S O(3,2)$ respec- 
tively $[34]^{10}$

$$
\begin{aligned}
& \chi_{4}\left(\Delta ; j_{1}, j_{2} \mid q, x, y\right)=\frac{q^{\Delta} f_{\mathrm{SU}(2)}\left(j_{1} \mid x\right) f_{\mathrm{SU}(2)}\left(j_{2} \mid y\right)}{\left(1-q x^{\frac{1}{2}} y^{\frac{1}{2}}\right)\left(1-q x^{\frac{1}{2}} y^{-\frac{1}{2}}\right)\left(1-q x^{-\frac{1}{2}} y^{\frac{1}{2}}\right)\left(1-q x^{-\frac{1}{2}} y^{-\frac{1}{2}}\right)}, \\
& \chi_{3}(\Delta ; j \mid q, x)=\frac{q^{\Delta} f_{\mathrm{SU}(2)}(j \mid x)}{(1-q)(1-q x)\left(1-q x^{-1}\right)}, \quad f_{\mathrm{SU}(2)}(j \mid x) \equiv \frac{x^{j+\frac{1}{2}}-x^{-j-\frac{1}{2}}}{x^{\frac{1}{2}}-x^{-\frac{1}{2}}} .
\end{aligned}
$$

Let us generalize (4.2) and define

$$
\chi\left(\operatorname{AdS}_{5} \mid q, x\right) \equiv \chi_{4}\left(\Delta_{5} ; \frac{s}{2}, \frac{s}{2} \mid q, x, x\right), \quad \chi\left(\operatorname{AdS}_{4} \mid q, x\right) \equiv \sum_{s^{\prime}=0}^{s} \sum_{k=0}^{\Delta_{5}-3} \chi_{3}\left(k+2 ; s^{\prime} \mid q, x\right)
$$

Let us also denote by tilde the "charge conjugation", i.e. the replacement $q \rightarrow q^{-1}, x \rightarrow$ $x^{-1}$. One can then check that

$$
\tilde{\chi}\left(\mathrm{AdS}_{5} \mid q, x\right)-\chi\left(\mathrm{AdS}_{5} \mid q, x\right)=\tilde{\chi}\left(\mathrm{AdS}_{4} \mid q, x\right)+\chi\left(\mathrm{AdS}_{4} \mid q, x\right) .
$$

This reduces to (4.4) in the "blind" limit $x \rightarrow 1$. The fact that (4.11) holds also for generic argument $x$ suggests that (4.4) has a group theoretic interpretation in terms of a map between representations of the corresponding $5 \mathrm{~d}$ and $4 \mathrm{~d}$ isometry groups.

\subsection{Relation between partition functions on $\mathrm{AdS}_{4}$ and $S^{1} \times S^{3}$}

As already mentioned above, given a conformal field in $\mathrm{AdS}_{d}$ we may make a Weyl transformation to replace $\mathrm{AdS}_{d}$ by half of the Einstein Universe $R \times S^{d-1}$ and then represent the partition function in $R \times S^{d-1}$ in terms of the partition function in $\mathrm{AdS}_{d}$ with two possible choices of boundary conditions. In the case of thermal quotients that leads to the relation (3.2).

In the special case of $\mathrm{AdS}_{4}$ it was observed in [4] that the two choices (+ and -) of the possible boundary conditions are equivalent, i.e. the corresponding higher spin representations are equivalent for $s>0$, with spin 0 (scalar) case being an exception. ${ }^{11}$ This suggests that for any conformal field not containing a scalar component we should have the equality between partition functions corresponding to the two alternative boundary conditions

$$
\begin{gathered}
\mathcal{Z}_{\mathrm{CF}}^{-}\left(\mathrm{AdS}_{4} ; q\right)=\mathcal{Z}_{\mathrm{CF}}^{+}\left(\mathrm{AdS}_{4} ; q\right) \\
\mathcal{Z}_{\mathrm{CF}}^{-}\left(\mathrm{AdS}_{4} ; q\right)=\widetilde{\mathcal{Z}}_{\mathrm{CF}}^{-}\left(\mathrm{AdS}_{4} ; q\right)+\sigma_{4}(q), \quad \widetilde{\mathcal{Z}}_{\mathrm{CF}}^{-}\left(\mathrm{AdS}_{4} ; q\right)=-\mathcal{Z}_{\mathrm{CF}}^{+}\left(\mathrm{AdS}_{4} ; q^{-1}\right)
\end{gathered}
$$

Then the relation (3.2) should simplify in $d=4$ case to

$$
\mathcal{Z}_{\mathrm{CF}}\left(S^{1} \times S^{3} ; q\right)=2 \mathcal{Z}_{\mathrm{CF}}^{+}\left(\mathrm{AdS}_{4} ; q\right)
$$

\footnotetext{
${ }^{10}$ Here $x$ and $y$ are chemical potentials for charges corresponding to other Cartan generators in addition to the dilatation operator.

${ }^{11}$ More generally, the fact that highest weight unitary representation of $\mathfrak{s o}(d, 2)$ algebra that admits extension to $\mathfrak{s o}(d+1,2)$ conformal algebra has two inequivalent extensions was demonstrated in [35]; for scalar field these representations are not equivalent as representations of $\mathfrak{s o}(d, 2)$ while for spin $s>0$ fields they are.
} 
This identity can be verified directly for the CHS or CST fields as follows.

The CHS partition function on $\mathrm{AdS}_{4}$ is a special case of $(3.14)^{12}$

$$
\mathrm{Z}_{\mathrm{CHS}, s}\left(\mathrm{AdS}_{4}\right)=\prod_{k=0}^{s-1}\left[\frac{\operatorname{det} \widehat{\Delta}_{k \perp}(k-(s-1)(s+2))}{\operatorname{det} \widehat{\Delta}_{s \perp}(s-(k-1)(k+2))}\right]^{1 / 2} .
$$

From (2.2) we see that the fields corresponding to terms in the numerator have $\Delta_{k}^{+}=s+2$, while the terms in the denominator give $\Delta_{k}^{+}=k+2$. The corresponding one-particle partition function is then a $d=4$ case of (3.17)

$$
\mathcal{Z}_{\mathrm{CHS}, s}^{+}\left(\mathrm{AdS}_{4} ; q\right)=\sum_{k=0}^{s-1} \frac{(2 s+1) q^{k+2}-(2 k+1) q^{s+2}}{(1-q)^{3}}=\frac{(2 s+1) q^{2}-(s+1)^{2} q^{s+2}+s^{2} q^{s+3}}{(1-q)^{4}}
$$

Comparing this to the CHS partition function on $S^{1} \times S^{3}$ given in Eq. (4.8) of [1] we find that indeed

$$
\mathcal{Z}_{\mathrm{CHS}, s}\left(S^{1} \times S^{3} ; q\right)=2 \mathcal{Z}_{\mathrm{CHS}, s}^{+}\left(\mathrm{AdS}_{4} ; q\right)
$$

In the case of the CST field the partition function on $S^{1} \times S^{3}$ was found in [28]. Comparing to (3.24) we conclude again that

$$
\mathcal{Z}_{\mathrm{CST}, s}\left(S^{1} \times S^{3} ; q\right)=2 \mathcal{Z}_{\mathrm{CST}, s}^{+}\left(\mathrm{AdS}_{4} ; q\right)
$$

The $4 \mathrm{~d}$ relation (4.14) may be extended also to the fermionic CHS fields, see Appendix (D).

This relation (4.14) is not, however, true for a conformal scalar and thus also for any conformal theory with $\mathrm{AdS}_{4}$ partition function containing a conformal scalar factor. In particular, it is not true for the 4-derivative conformal scalar field as follows from the comparison of (C.12) and (C.8) in Appendix C. Another counter-example is the conformal theory of an antisymmetric rank 2 tensor discussed in Appendix E. ${ }^{13}$

\subsection{Further descent: AdS $_{4} \rightarrow \mathbb{R} \times S^{2}$}

Given a conformal field in $4 \mathrm{~d}$ related to some higher spin field in $\mathrm{AdS}_{5}$ we may define it on $\mathrm{AdS}_{4}$ and then further associate to it another conformal field $\widehat{\mathrm{CF}}$ at the $\mathrm{AdS}_{4}$ boundary $\mathbb{R} \times S^{2}$. This gives a triplet of fields

$$
\mathrm{HS} \text { on } \mathrm{AdS}_{5} \longrightarrow \mathrm{CF} \text { on } \mathrm{AdS}_{4} \longrightarrow \widehat{\mathrm{CF}} \text { on } \mathbb{R} \times S^{2} .
$$

Then the thermal partition functions of $\mathrm{CF}$ and $\widehat{\mathrm{CF}}$ are related by (1.3) or (2.7), i.e. for the second step we get

$$
\mathcal{Z}_{\mathrm{CF}}^{-}\left(\mathrm{AdS}_{4} ; q\right)-\mathcal{Z}_{\mathrm{CF}}^{+}\left(\mathrm{AdS}_{4} ; q\right)=\mathcal{Z}_{\widehat{\mathrm{CF}}}\left(S^{1} \times S^{2} ; q\right)
$$

Combining this with (4.12) we conclude that $\widehat{\mathrm{CF}}$ should have zero partition function,

$$
\mathcal{Z}_{\widehat{\mathrm{CF}}}\left(S^{1} \times S^{2} ; q\right)=0
$$

\footnotetext{
${ }^{12}$ While here we have a scalar field contribution at $k=0$ this is not a conformal massless scalar but a ghost field needed to guarantee the conformal invariance of the spin s CHS field.

${ }^{13}$ Here the presence of a scalar components is apparent also in the approach developed in [36].
} 
Then the total partition function of the theory defined on "boundary of the boundary" is $Z=1$, i.e. the resulting $3 \mathrm{~d}$ conformal theory $\widehat{\mathrm{CF}}$ should be "trivial" or "topological". We will also define $\widehat{\mathrm{CF}}$ on $\mathrm{AdS}_{3}$ and then find, in agreement with (3.3), that

$$
\mathcal{Z}_{\widehat{\mathrm{CF}}}^{+}\left(\mathrm{AdS}_{3} ; q\right)=\mathcal{Z}_{\widehat{\mathrm{CF}}}^{-}\left(\mathrm{AdS}_{3} ; q\right)=0 .
$$

Before giving some explicit examples let us first recall that a totally symmetric spin $s$ CHS field $\phi_{s}$ in $d$ dimensions has the action

$$
S_{\mathrm{CHS}_{s}}=\int d^{d} x \phi_{s} P_{s} \partial^{2 s+d-4} \phi_{s}=\int d^{d} x C_{s} \partial^{d-4} C_{s},
$$

where $P_{s}$ is a projector onto transverse traceless tensors and $C_{s} \sim \partial^{s} \phi_{s}$ is gauge-invariant field strength (generalized Maxwell or Weyl tensor). The number of the corresponding dynamical degrees of freedom is $[16,21]$

$$
v_{s, d}=\frac{(d-3)(2 s+d-2)(2 s+d-4)(s+d-4) !}{2(d-2) ! s !} .
$$

Eq. (4.22) is local for even $d \geq 4$ (where $\partial^{2}=\square$ enters in positive power) but can be formally defined also for odd $d$. The case of $d=3$ is special in that the number of dynamical degrees of freedom (4.23) vanishes, while (4.22) takes a non-local form

$$
S_{\mathrm{CHS}_{s}}=\int d^{3} x \phi_{s} P_{s} \square^{s-1 / 2} \phi_{s}=\int d^{3} x C_{s} \square^{-1 / 2} C_{s} .
$$

Let us also recall that the CHS action in $d$ dimensions may be viewed as an induced one [37] from a free $\mathrm{CFT}_{d}$ : if $\phi_{s}$ is coupled to a spin-s conserved current $J_{s}$ then the kinetic term of $\phi_{s}$ is determined by the 2-point function $\left\langle J_{s}(x) J_{s}\left(x^{\prime}\right)\right\rangle \cdot{ }^{14}$ Insisting on locality one may consider a Chern-Simons type action for the corresponding $3 \mathrm{~d}$ CHS field that may be induced from chiral $3 \mathrm{~d}$ fermions (see [40-42] and [43-45] for $s=2$ ). For a more natural parity-even case induced from a free $3 \mathrm{~d}$ scalar CFT we get in momentum space $\left\langle J_{s} J_{s}\right\rangle=\frac{k_{s}}{\sqrt{p^{2}}} \tilde{P}_{s}(p)$, where $\tilde{P}_{s}(p)$ is Fourier transform of the transverse traceless projector in (4.22) (i.e. a symmetrized and traceless product of $s$ factors of $\left.\left(\tilde{P}_{1}\right)_{\mu}^{v}=\delta_{\mu}^{v}-\frac{p_{\mu} p^{v}}{p^{2}}\right)$. The corresponding parity even $3 \mathrm{~d}$ CHS action is then given by (4.22).

\subsubsection{Spin 1}

Let us now illustrate (4.20) turn to some special cases and start with a massless spin 1 gauge field in $\mathrm{AdS}_{5}$ that has $\Delta_{5}^{+}=2+s=3$ and is associated with the following combination of $S O(2,4)$ representations $\mathrm{MHS}_{1}\left(\mathrm{AdS}_{5}\right)=\left(3 ; \frac{1}{2}, \frac{1}{2}\right)-(4 ; 0,0)$. The corresponding $4 \mathrm{~d}$ boundary field is the $s=1 \mathrm{CHS}$ field, i.e. the standard Maxwell theory (cf. (4.22)). Its partition function when defined on $\mathrm{AdS}_{4}$ is a special case of (3.14)

$$
Z_{\mathrm{CHS}, 1}\left(\mathrm{AdS}_{4}\right)=\left[\frac{\operatorname{det} \widehat{\Delta}_{0}(0)}{\operatorname{det} \widehat{\Delta}_{1 \perp}(3)}\right]^{1 / 2} .
$$

\footnotetext{
${ }^{14}$ In general, in $3 \mathrm{~d}$ there are two possible conformally invariant tensor structures that may appear in a two-point function of a conserved current $J_{s}$ : a non-local parity-even and a local parity-odd one (see, e.g., $[38,39,2])$.
} 
Here each operator is in turn associated with a conformal field at the $R \times S^{2}$ boundary (we get a scalar with $\Delta_{4}^{+}=3$ and a transverse vector with $\Delta_{4}^{+}=2$ ). The corresponding combination of $S O(2,3)$ representations $\left(\Delta_{4}^{+} ; j\right)$ is ${ }^{15}$

$$
\mathrm{CHS}_{1}\left(\mathrm{AdS}_{4}\right)=(2 ; 1)-(3 ; 0)=\mathrm{MHS}_{1}\left(\mathrm{AdS}_{4}\right) \text {. }
$$

Thus the field at the $3 \mathrm{~d}$ boundary should be the $s=1$ member of the $3 \mathrm{~d}$ CHS family (4.24) with a non-local action (cf. [46, 2])

$$
S_{\mathrm{CHS}_{1}}=\int d^{3} x F_{\mu v} \square^{-1 / 2} F_{\mu \nu} .
$$

This theory is effectively topological, having no dynamical degrees of freedom (in agreement with (4.23)). One can see this explicitly, e.g., by computing the corresponding partition function in flat $3 \mathrm{~d}$ space ${ }^{16}$

$$
\mathrm{Z}_{\mathrm{CHS}_{1}}\left(\mathbb{R}^{3}\right)=\left[\frac{\operatorname{det} \square}{\operatorname{det}\left(\partial \square^{-1 / 2} \partial\right)_{1 \perp}}\right]^{1 / 2}=\left[\frac{\operatorname{det} \square}{\operatorname{det}\left(\square^{1 / 2}\right)_{1 \perp}}\right]^{1 / 2} \stackrel{3 \mathrm{~d}}{=}\left[\frac{\operatorname{det} \square}{\operatorname{det}\left(\square^{1 / 2}\right)^{2}}\right]^{1 / 2}=1 .
$$

As there is no conformal anomaly in odd dimensions the same should be true also for all conformally flat spaces, e.g., $\mathrm{AdS}_{3}$

$$
\mathrm{Z}_{\mathrm{CHS}_{1}}\left(\mathrm{AdS}_{3}\right)=1
$$

Defining the 4d Maxwell field on $\mathrm{AdS}_{4}$ we get from (A.6)

$$
\begin{aligned}
\widetilde{\mathcal{Z}}_{\mathrm{CHS}_{1}}^{-}\left(\mathrm{AdS}_{4} ; q\right)-\mathcal{Z}_{\mathrm{CHS}_{1}}^{+}\left(\mathrm{AdS}_{4} ; q\right) & =-\mathcal{Z}_{\mathrm{CHS}_{1}}^{+}\left(\mathrm{AdS}_{4} ; q^{-1}\right)-\mathcal{Z}_{\mathrm{CHS}_{1}}^{+}\left(\mathrm{AdS}_{4} ; q\right) \\
& =-\frac{3 / q^{2}-1 / q^{3}}{(1-1 / q)^{3}}-\frac{3 q^{2}-q^{3}}{(1-q)^{3}}=-1
\end{aligned}
$$

This -1 is precisely what is removed by the $\sigma_{\mathrm{CHs}_{1,4}}(q)$ term in (2.6) in agreement with (4.12) so that we get $\mathcal{Z}_{\mathrm{CHS}_{1}}\left(S^{1} \times S^{2} ; q\right)=0$ as a special case of $(4.20) .{ }^{17}$

We can also explicitly check the equality (3.4) of the $\sigma$-terms. For the $\mathrm{MHS}_{s}\left(\mathrm{AdS}_{5}\right)$ theory the $\sigma_{\mathrm{MHS}, 5}(q)$ term for a general spin $s$ may be found in Eq. (5.5) of [1] and for $s=1$ it is equal to 1 , i.e. is indeed the same as the above $\sigma_{\mathrm{CHS}_{1}, 4}(q)$.

\footnotetext{
${ }^{15}$ Here $j$ is the $S O(3)$ angular momentum. In general, $\mathrm{MHS}_{s}\left(\mathrm{AdS}_{4}\right)=(1+s ; s)-(2+s ; s-1)$, i.e. it corresponds to a spin $s$ field with gauge invariance with spin $s-1$ parameter. The partition function for a massive $S O(2,3)$ representation $\left(\Delta_{4} ; s\right)$ is given by $\mathcal{Z}_{\left(\Delta_{4} ; s\right)}^{+}(q)=(2 s+1) \frac{q^{\Delta_{4}}}{(1-q)^{3}}$.

${ }^{16}$ Here the measure contribution from the decomposition $A_{\mu}=A_{\mu \perp}+\partial_{\mu} \phi$ cancels against the kinetic operator contribution. Note also that in $3 \mathrm{~d}$ one can dualize $F_{\mu v} \frac{1}{\square^{1 / 2}} F_{\mu \nu}$ to a scalar with kinetic term $\phi \square^{3 / 2} \phi$ but the corresponding partition function is still 1 as the scalar determinant is cancelled by the measure contribution coping from integrating out the auxiliary field $F_{\mu \nu}$.

${ }^{17}$ Indeed, the partition function on $S^{1} \times S^{d-1}$ for the $\mathrm{CHS}_{1}$ Maxwell field with the action in (4.22), i.e. $\int d^{d} x F^{\mu v} \square^{\frac{d-4}{2}} F_{\mu \nu}$, was already computed in [1] with the general expression being

$$
\mathcal{Z}_{\mathrm{CHS}_{1}}\left(S^{1} \times S^{d-1} ; q\right)=1-\frac{1-d q+d q^{d-1}-q^{d}}{(1-q)^{d}}
$$
}

This vanishes for $d=3$. 


\subsubsection{Spin 2}

Let us now consider the $s=2$ case, i.e. start with the MHS theory in $\mathrm{AdS}_{5}$ describing massless rank-2 tensor with $\Delta_{5}^{+}=2+s=4$ and spin 1 gauge invariance parameter, i.e. associated with the following combination of $S O(2,4)$ representations $\mathrm{MHS}_{2}\left(\mathrm{AdS}_{5}\right)=$ $(4 ; 1,1)-\left(5 ; \frac{1}{2}, \frac{1}{2}\right)$. The dual conformal field in $4 \mathrm{~d}$ is the $s=2$ CHS theory, i.e. Weyl gravity. Its partition function on $\mathrm{AdS}_{4}$ is a special case of $(3.23)$, i.e. [47, 48, 29]

$$
\mathrm{Z}_{\mathrm{CHS}_{2}}\left(\mathrm{AdS}_{4}\right)=\left[\frac{\operatorname{det} \widehat{\Delta}_{0}(-4) \operatorname{det} \widehat{\Delta}_{1 \perp}(-3)}{\operatorname{det} \widehat{\Delta}_{2 \perp}(4) \operatorname{det} \widehat{\Delta}_{2 \perp}(2)}\right]^{1 / 2} .
$$

Using (2.2) the values of the scaling dimensions $\Delta_{4}^{+}$corresponding to each factor in (4.31) are (cf. (2.1))

$$
\begin{array}{|c|cccc|}
\hline & \widehat{\Delta}_{2 \perp}(4) & \widehat{\Delta}_{2 \perp}(2) & \widehat{\Delta}_{1 \perp}(-3) & \widehat{\Delta}_{0}(-4) \\
\hline \Delta_{4}^{+} & 2 & 3 & 4 & 4 \\
\hline
\end{array}
$$

This means that the equivalent combination of $S O(2,3)$ representations is ${ }^{18}$

$$
\mathrm{CHS}_{2}\left(\mathrm{AdS}_{4}\right)=\mathrm{MHS}_{2}\left(\mathrm{AdS}_{4}\right) \oplus \mathrm{PM}_{2}^{(2)}\left(\mathrm{AdS}_{4}\right)=[(3 ; 2)-(4 ; 1)] \oplus[(2 ; 2)-(4 ; 0)] \text {. }
$$

Indeed, the Weyl graviton on $\mathrm{AdS}_{4}$ is a combination of Einstein graviton and a partially massless spin 2 field with scalar gauge invariance $[49,50,20]$.

The $3 \mathrm{~d}$ conformal theory "induced" by $4 \mathrm{~d}$ Weyl graviton at the boundary of $\mathrm{AdS}_{4}$ thus contains two parts. From the Einstein graviton $\mathrm{MHS}_{2}\left(\mathrm{AdS}_{4}\right)$ we get a conformally invariant $3 \mathrm{~d} \mathrm{CHS}_{2}$ or Weyl theory with parity-even non-local linearized action (4.24), i.e. $\int d^{3} x C_{2} \square^{-1 / 2} C_{2}$ (see also [39]). ${ }^{19}$ From the partially massless field $\mathrm{PM}_{2}^{(2)}\left(\mathrm{AdS}_{4}\right)$ we get $\mathrm{CST}_{2}$ field representing a non-unitary $3 \mathrm{~d}$ symmetric tensor $\varphi_{\mu v}$ with scalar gauge invariance $\delta \varphi_{\mu \nu}=\partial_{\mu} \partial_{\nu} \epsilon$. This theory has a non-local action $\int d^{3} x \varphi_{2} \mathrm{P}_{2} \square^{1 / 2} \varphi_{2}$ where $\mathrm{P}_{2}$ is an appropriate projector ensuring scalar gauge invariance. ${ }^{20}$ In summary, the conformal field combination corresponding to (4.33) on flat $3 \mathrm{~d}$ boundary is ${ }^{21}$

$$
\widehat{\mathrm{CF}}\left(\mathbb{R}^{3}\right)=\mathrm{CHS}_{2}\left(\mathbb{R}^{3}\right) \oplus \mathrm{CST}_{2}\left(\mathbb{R}^{3}\right) .
$$

Let us now show that this system has zero total number $v$ of dynamical degrees of freedom. Indeed, the $\mathrm{CHS}_{2}$ field has $v=0$ according to (4.23). For $\mathrm{CST}_{2}$ the flat space partition function is

$$
Z_{\mathrm{CST}_{2}}\left(\mathbb{R}^{3}\right)=\left[\frac{\operatorname{det} \square_{1 \perp}\left(\operatorname{det} \square_{0}\right)^{2}}{\left(\operatorname{det} \square^{1 / 2}\right)_{2 \perp}\left(\operatorname{det} \partial \square^{1 / 2} \partial\right)_{1 \perp}}\right]^{1 / 2}=\left[\frac{\operatorname{det} \square_{1 \perp}\left(\operatorname{det} \square_{0}\right)^{2}}{\left(\operatorname{det} \square_{2 \perp}\right)^{1 / 2}\left(\operatorname{det} \square_{1 \perp}\right)^{3 / 2}}\right]^{1 / 2}=1 .
$$

\footnotetext{
${ }^{18}$ In general, for a partially massless spin $s$ field we have $\mathrm{PM}_{s}^{(s)}\left(\mathrm{AdS}_{4}\right)=(2 ; s)-(2+s ; 0)$.

${ }^{19}$ The full non-linear action of Weyl-invariant gravity in $3 d$ with quadratic part given by $\int d^{3} x C_{2} \square^{-1 / 2} C_{2}$ where $C_{2}$ is linearized Weyl tensor can be obtained as an induced one corresponding to a conformally coupled scalar in 3 d, i.e. as $\log \operatorname{det}\left(-\nabla^{2}+\frac{1}{8} R\right)$. Since there is no Weyl anomaly in 3 dimensions this non-local functional of the metric will be both reparametrization and Weyl invariant.

${ }^{20}$ Indeed, from (4.33) we find that the canonical dimension of $\varphi_{\mu v}$ is $d-\Delta^{+}=3-2=1$.

${ }^{21}$ An alternative way of obtaining (quadratic) action for this set of $3 \mathrm{~d}$ fields is to start with the (linearized) Weyl gravity in $\mathrm{AdS}_{4}$ space, specify separate boundary conditions for the $4 \mathrm{~d}$ graviton and partially massless mode (in terms of $3 \mathrm{~d}$ graviton and $3 \mathrm{~d} \mathrm{CST}_{2}$ field respectively) and then evaluate the $4 \mathrm{~d}$ action on the solution of the equations of motion. This procedure may have non-linear generalization if one starts with the full non-linear $4 \mathrm{~d}$ Weyl gravity action and considers a generic asymptotically $\mathrm{AdS}_{4}$ background.
} 
Here the numerator is the Jacobian for the change of variables $\varphi_{\mu \nu}=\varphi_{\mu \nu}^{\perp}+\partial_{(\mu} V_{v)}^{\perp}+$ $\left(\partial_{\mu} \partial_{\nu}-\frac{1}{3} \delta_{\mu v} \partial^{2}\right) \gamma$. The denominator is from the action $\int d^{3} x \varphi_{2} \mathrm{P}_{2} \square^{1 / 2} \varphi_{2}$ where the scalar component $\gamma$ drops out due to gauge invariance. Thus $\left.v\left(\mathrm{CST}_{2}\right)\right|_{d=3}=\frac{3}{2} \times 2+\frac{1}{2} \times 2-$ $(2+2)=0$. We have used that in three dimensions a contribution $\left(\operatorname{det} \square_{s \perp}\right)^{-1 / 2}$ in the partition function is equivalent ${ }^{22}$ to $\left(\operatorname{det} \square_{0}\right)^{-1}$ (i.e. $v=2$ ) for $s>0$.

Let us note for completeness that (4.35) may be generalized to any spin $s>0$ as follows

$$
Z_{\mathrm{CST}_{s}}\left(\mathbb{R}^{3}\right)=\left[\frac{\operatorname{det} \square_{s-1, \perp} \operatorname{det} \square_{s-2, \perp}^{2} \cdots \operatorname{det} \square^{s}}{\operatorname{det} \square_{s \perp}^{1 / 2} \operatorname{det} \square_{s-1, \perp}^{3 / 2} \cdots \operatorname{det} \square_{1 \perp}^{s-1 / 2}}\right]^{1 / 2}=1 .
$$

Thus as for a CHS field, the total number of d.o.f. of a 3d CST field is zero for any $s$ : $v=2 \sum_{n=1}^{s}\left(n-\frac{1}{2}\right)-\left(2 \sum_{n=1}^{s-1} n+s\right)=0$.

At the level of partition function, the decomposition (4.33) implies

$$
\mathcal{Z}_{\mathrm{CHS}_{2}}^{+}\left(\mathrm{AdS}_{4} ; q\right)=\frac{5 q^{3}-3 q^{4}}{(1-q)^{3}}+\frac{5 q^{2}-q^{4}}{(1-q)^{3}}=\frac{q^{2}\left(5+5 q-4 q^{2}\right)}{(1-q)^{3}}
$$

We may compute the partition function of $\widehat{\mathrm{CF}}=\mathrm{CHS}_{2} \oplus \mathrm{CST}_{2}$ as in (2.7),(2.6)

$$
\begin{aligned}
\mathcal{Z}_{\widehat{\mathrm{CF}}}\left(S^{1} \times S^{2} ; q\right) & =-\mathcal{Z}_{\mathrm{CHS}_{2}}^{+}\left(\mathrm{AdS}_{4} ; q^{-1}\right)-\mathcal{Z}_{\mathrm{CHS}_{2}}^{+}\left(\operatorname{AdS}_{4} ; q\right)+\sigma_{\mathrm{CHS}_{2}, 4}(q) \\
& =-4\left(q+q^{-1}\right)-7+\sigma_{\mathrm{CHS}_{2}, 4}(q)=0
\end{aligned}
$$

which is in agreement with (4.20) (see also Appendix B). We used that, as one can check, $\sigma_{\mathrm{CHS}_{2}, 4}(q)=4\left(q+q^{-1}\right)+7.23$ Again, we can compare the expression for $\sigma_{\mathrm{CHS}_{2,4}}(q)$ with the $\sigma_{\mathrm{MHS}_{2,5}}(q)$ term in Eq. (5.5) of [1] for $s=2$ and thus verify the relation (3.4).

\section{Concluding remarks}

The "(AdS/CFT $)^{2}=0$ " relation (4.20) is special to $d=4$ case because we used (4.12) to obtain it. In $d>4$ case we expect to find a more complicated picture. For example, suppose we start with an massless HS field in $\mathrm{AdS}_{7}$. Then at the boundary we get conformal HS field in $6 \mathrm{~d}$ and can define it on $\mathrm{AdS}_{6}$ thus associating to it some other conformal field $\mathrm{CF}_{5}$ at the boundary of $\mathrm{AdS}_{6}$. We can then continue the descent, i.e. define $\mathrm{CF}_{5}$ on $\mathrm{AdS}_{5}$ and associate to it another conformal field $\mathrm{CF}_{4}$ at the boundary of $\mathrm{AdS}_{5}$, etc. One may then look for some new identities between partition functions of these fields in addition to (2.7) and (3.1). For example, one can check that in the spin 1 case $\mathrm{CF}_{5}$ appears to be represented by a combination of $5 \mathrm{~d} \mathrm{CHS}_{1}$ field (with non-local action $\int d^{5} x F_{\mu v} \square^{1 / 2} F_{\mu \nu}$ as in (4.22)) and an extra field, such that one ends up with $\mathrm{CF}_{4}$ being just the standard $\mathrm{CHS}_{1}$ Maxwell field. Details of the corresponding relations between partition functions remain to be studied.

\footnotetext{
${ }^{22}$ The number of components of a totally symmetric traceless rank-s tensor $\phi_{s}$ in $d$ dimensions is $N_{S}=$ $\left(\begin{array}{c}s+d-1 \\ s\end{array}\right)-\left(\begin{array}{c}s+d-3 \\ s-2\end{array}\right)$, i.e. $\left.N_{s}\right|_{d=3}=2 s+1$. The number of components of transverse $\left(\partial \cdot \phi_{s \perp}=0\right)$ traceless rank $s>0$ tensor is $N_{s, \perp}=N_{s}-N_{s-1} \stackrel{3 d}{\rightarrow} 2$.

${ }^{23}$ Here we can use the expression for $\mathcal{Z}_{\mathrm{CHS}_{2}}\left(S^{1} \times S^{d-1} ; q\right)$ in Eq. (5.17) of [1] and check that it vanishes for $d=3$.
} 
Given the general relations (1.3) and (1.8) between partition functions of particular higher spin fields one may apply them to theories containing infinite number of spins. For example, the Vasiliev-type theory in $\mathrm{AdS}_{d+1}$ (containing a scalar and totally symmetric MHS spin $1,2, \ldots$ fields and dual to the singlet sector of free $U(N)$ scalar theory in $4 \mathrm{~d}$ ) is naturally associated to the CHS theory of all conformal spins $s=0,1,2, \ldots$ in $d$ dimensions (with linearized action (4.22)). Summing over all spins the relation (1.8) should trivialise. Indeed, for the MHS theory we find [26] (spin 0 field here has $\Delta^{+}=d-2$ )

$$
\begin{aligned}
& \mathcal{Z}_{\mathrm{MHS}}^{+}\left(\operatorname{AdS}_{d+1} ; q\right)=\sum_{s=0}^{\infty} \mathcal{Z}_{\mathrm{MHS}, \mathrm{S}}^{+}\left(\operatorname{AdS}_{d+1} ; q\right)=\frac{q^{d-2}(1+q)^{2}}{(1-q)^{2 d-2}} \\
& \widetilde{\mathcal{Z}}_{\mathrm{MHS}}^{-}\left(\operatorname{AdS}_{d+1} ; q\right)=(-1)^{d} \mathcal{Z}_{\mathrm{MHS}}^{+}\left(\operatorname{AdS}_{d+1} ; q^{-1}\right)=(-1)^{d} \mathcal{Z}_{\mathrm{MHS}}^{+}\left(\operatorname{AdS}_{d+1} ; q\right) .
\end{aligned}
$$

Thus, e.g., for $d=4$ the l.h.s. of (1.8) vanishes after summing over all spins (the $\sigma$ term in $\mathcal{Z}^{-}$in (1.5) drops out being symmetric under $q \rightarrow q^{-1}$ ). At the same time, the summed CHS partition function on thermal $\mathrm{AdS}_{d}$ in (3.17) appears to be divergent (cf. [1]). In four dimensions $\mathcal{Z}_{\mathrm{CHS}, s}^{+}\left(\mathrm{AdS}_{4} ; q\right)$ is given by (4.16) and the divergence is due to the term $\sim(2 s+1) q^{2}$ in the numerator that is not suppressed at large $s$. Nevertheless, applying the analog of the standard $\zeta$-function regularization (i.e. $\sum_{s=1}^{\infty} 1=\zeta(0)=-\frac{1}{2}$, etc.), we obtain for the regularized expression of the sum over spins

$$
\begin{aligned}
& \mathcal{Z}_{\mathrm{CHS}}^{+}\left(\mathrm{AdS}_{4} ; q\right)=\lim _{z \rightarrow 0} \sum_{s=1}^{\infty} s^{z} \mathcal{Z}_{\mathrm{CHS}, \mathrm{s}}^{+}\left(\operatorname{AdS}_{4} ; q\right)=-\frac{2}{3} \frac{q^{2}\left(q^{2}+4 q+1\right)}{(1-q)^{6}} \\
& \mathcal{Z}_{\mathrm{CHS}}^{+}\left(\mathrm{AdS}_{4} ; q\right)=\mathcal{Z}_{\mathrm{CHS}}^{+}\left(\mathrm{AdS}_{4} ; q^{-1}\right), \quad \widetilde{\mathcal{Z}}_{\mathrm{CHS}}^{-}\left(\operatorname{AdS}_{4} ; q\right)=-\mathcal{Z}_{\mathrm{CHS}}^{+}\left(\operatorname{AdS}_{4} ; q\right) .
\end{aligned}
$$

Thus the r.h.s. of (1.8) also vanishes. The same conclusion is reached also in general even dimension $d>4$ once the non-trivial spin 0 contribution is included in the sum in (5.3).

\section{Acknowledgments}

We thank M. Grigoriev, I. Lovrekovic and R. Metsaev for useful discussions. The work of AAT was supported by the ERC Advanced grant No.290456, the STFC Consolidated grant ST/L00044X/1 and by the Russian Science Foundation grant 14-42-00047 associated with Lebedev Institute.

\section{A Reconstructing $\mathcal{Z}_{\mathrm{CF}}^{+}\left(\mathrm{AdS}_{d}\right)$ from $\mathcal{Z}_{\mathrm{HS}}^{+}\left(\operatorname{AdS}_{d+1}\right)$}

Here we shall reverse the logic: assume that the relation (3.5) is true and use it to determine the partition function $\mathcal{Z}_{\mathrm{CF}}^{+}\left(\mathrm{AdS}_{d}\right)$ from the knowledge of $\mathcal{Z}_{\mathrm{HS}}^{+}\left(\mathrm{AdS}_{d+1}\right)$ just by doing algebraic manipulations.

These partition functions have the following general form

$$
\mathcal{Z}_{\mathrm{HS}}^{+}\left(\mathrm{AdS}_{d+1} ; q\right)=\frac{P(q) q^{\frac{d}{2}}}{(1-q)^{d}}, \quad \mathcal{Z}_{\mathrm{CF}}^{+}\left(\mathrm{AdS}_{d} ; q\right)=\frac{F(q) q^{\frac{d-1}{2}}}{(1-q)^{d-1}}
$$


where $P(q)$ and $F(q)$ are finite sums of non-negative powers of $q$ (in $\operatorname{AdS}_{d}$ we have $\Delta_{d}^{+} \geq$ $\frac{1}{2}(d-1)$, cf. (2.3)). Eq. (3.5) or (3.6) implies that

$$
F(q)+F\left(q^{-1}\right)=\frac{\sqrt{q}}{1-q}\left[P\left(q^{-1}\right)-P(q)\right] .
$$

The r.h.s. of (A.2) may be expanded in a Laurent series around $q=0$ and comparing with the 1.h.s., we may then determine $F(q)$.

Let us consider some examples. Let us start with the conformal scalar in $\mathrm{AdS}_{4}$ which corresponds to a massive scalar in $\mathrm{AdS}_{5}$ with $\Delta_{5}^{+}=3$, i.e. (cf. (3.7))

$$
\mathcal{Z}_{0}^{+}\left(\mathrm{AdS}_{5} ; q\right)=\frac{q^{3}}{(1-q)^{4}}, \quad P(q)=q .
$$

Then (A.2) gives

$$
F(q)+F\left(q^{-1}\right)=\frac{q+1}{\sqrt{q}}=q^{-1 / 2}+q^{1 / 2} \quad \longrightarrow \quad F(q)=q^{1 / 2}
$$

and therefore, in agreement with (3.10), $\mathcal{Z}_{\mathrm{cs}}^{+}\left(\mathrm{AdS}_{4} ; q\right)=\frac{q^{2}}{(1-q)^{3}}$.

Another example is the spin 1 field in $\mathrm{AdS}_{5}$ corresponding to spin $1 \mathrm{CHS}$ field (i.e. Maxwell field) in $\mathrm{AdS}_{4}$. From (3.19) we have

$$
\mathcal{Z}_{\mathrm{MHS}_{1}}^{+}\left(\mathrm{AdS}_{5} ; q\right)=\frac{4 q^{3}-q^{4}}{(1-q)^{4}}, \quad P(q)=4 q-q^{2}
$$

Then, (A.2) gives

$$
F(q)+F\left(q^{-1}\right)=-q^{3 / 2}-q^{-3 / 2}+3 q^{1 / 2}+3 q^{-1 / 2} \quad \longrightarrow \quad F(q)=3 q^{1 / 2}-q^{3 / 2},
$$

and therefore we get, in agreement with (3.17), $\mathcal{Z}_{\mathrm{CHS}_{1}}^{+}\left(\mathrm{AdS}_{4} ; q\right)=\frac{3 q^{2}-q^{3}}{(1-q)^{3}}$.

Our third example is a non-unitary CFT represented by a vector $V_{\mu}$ in $6 \mathrm{~d}$ with 2 ndderivative kinetic term. This is a special $s=1$ case of CST family of conformal fields described by rank-s symmetric tensors $\varphi_{\mu_{1} \ldots \mu_{s}}$ which in $d=6$ have no gauge invariance. As discussed in [33], this CF is induced by a higher spin field in $\mathrm{AdS}_{7}$ transforming in the $\left(\Delta ; h_{1}, h_{2}, h_{3}\right)=(4 ; 1,0,0)$ representation of $S O(2,6)$. Taking into account that $\operatorname{dim}[1,0,0]=6$, we get (cf. (2.3))

$$
\mathcal{Z}_{\mathrm{HS}}^{+}\left(\mathrm{AdS}_{7} ; q\right)=\frac{6 q^{4}}{(1-q)^{6}}, \quad P(q)=q .
$$

Then from (A.2) we have again $F(q)=q^{1 / 2}$ as in (A.4) and thus we predict that

$$
\mathcal{Z}_{V}^{+}\left(\operatorname{AdS}_{6} ; q\right)=\frac{6 q^{3}}{(1-q)^{5}}
$$

This expression follows indeed from the general expression for the $V_{\mu}$ partition function on $S^{6}$ or on $\mathrm{AdS}_{6}$ given in Eq. (A.4) of [33]

$$
Z_{V}\left(\operatorname{AdS}_{6}\right)=\left[\operatorname{det} \widehat{\Delta}_{1 \perp}(7) \operatorname{det} \widehat{\Delta}_{0}(6)\right]^{-1 / 2}
$$

Applying (2.2) to the operators here we find that we have the same $\Delta_{6}^{+}=3$ and $\Delta_{6}^{-}=2$ for both factors. However, the number of degrees of freedom of a transverse vector in $6 \mathrm{~d}$ is $6-1=5$ while the scalar contributes only one. Thus the numerator of $\mathcal{Z}_{V}^{+}\left(\operatorname{AdS}_{6} ; q\right)$ should be $(5+1) q^{3}=6 q^{3}$, in agreement with (A.8). 


\section{B $\sigma$-term relation in Eq. (3.4)}

Let us now use the general general structure (A.1),(A.2) of the higher spin partition functions in $\mathrm{AdS}_{d+1}$ and the corresponding conformal field partition functions in $\mathrm{AdS}_{d}$ to justify the equality in (3.4).

According to (2.6),(2.7) we have for the partition function of CF on $S^{1} \times S^{d}$ and the partition of another conformal field $\widehat{\mathrm{CF}}$ (the one associated to $\mathrm{CF}$ on $\mathrm{AdS}_{d}$ ) on $S^{1} \times S^{d-1}$

$$
\begin{aligned}
& \mathcal{Z}_{\mathrm{CF}}\left(S^{1} \times S^{d} ; q\right)=\widetilde{\mathcal{Z}}_{\mathrm{HS}}^{-}\left(\operatorname{AdS}_{d+1} ; q\right)-\mathcal{Z}_{\mathrm{HS}}^{+}\left(\operatorname{AdS}_{d+1} ; q\right)+\sigma_{\mathrm{HS}, d+1}(q), \\
& \mathcal{Z}_{\widehat{\mathrm{CF}}}\left(S^{1} \times S^{d-1} ; q\right)=\widetilde{\mathcal{Z}}_{\mathrm{CF}}^{-}\left(\operatorname{AdS}_{d} ; q\right)-\mathcal{Z}_{\mathrm{CF}}^{+}\left(\operatorname{AdS}_{d} ; q\right)+\sigma_{\mathrm{CF}, d}(q) .
\end{aligned}
$$

Using $\widetilde{\mathcal{Z}}^{-}\left(\operatorname{AdS}_{n} ; q\right)=(-1)^{n+1} \mathcal{Z}^{+}\left(\operatorname{AdS}_{n} ; q^{-1}\right)$, and also (A.2), we find

$$
\begin{aligned}
& \mathcal{Z}_{\mathrm{CF}}\left(S^{1} \times S^{d} ; q\right)=\frac{q^{\frac{d-1}{2}}\left[F\left(q^{-1}\right)+F(q)\right]}{(1-q)^{d-1}}+\sigma_{\mathrm{HS}, d+1}(q), \\
& \mathcal{Z}_{\widehat{\mathrm{CF}}}\left(S^{1} \times S^{d-1} ; q\right)=\frac{q^{\frac{d-1}{2}}\left[F\left(q^{-1}\right)-F(q)\right]}{(1-q)^{d-1}}+\sigma_{\mathrm{CF}, d}(q) .
\end{aligned}
$$

The role of the $\sigma$-terms is to remove the negative powers of $q$ in the expansion around $q=0$ of the r.h.s. of (B.3),(B.4) as such terms cannot be present on the 1.h.s. that can be computed using operator counting method and thus should have only positive powers of q. Such terms come only from the $F\left(q^{-1}\right)$ term that is the same in the two lines of (B.3). This then implies that

$$
\sigma_{\mathrm{HS}, d+1}(q)=\sigma_{\mathrm{CF}, d}(q) .
$$

To give an example, let us consider the $\mathrm{CHS}_{2}$ field in $4 \mathrm{~d}$ for which from (4.37) we have

$$
\mathcal{Z}_{\mathrm{CHS}, 2}^{+}\left(\mathrm{AdS}_{4} ; q\right)=\frac{q^{2}\left(5+5 q-4 q^{2}\right)}{(1-q)^{3}} \longrightarrow F(q)=5 q^{1 / 2}+5 q^{3 / 2}-4 q^{5 / 2}
$$

Then (B.3) contains

$$
\frac{q^{3 / 2} F\left(q^{-1}\right)}{(1-q)^{3}}=\frac{-4+5 q+5 q^{2}}{q(1-q)^{3}}=-4 q^{-1}-7-4 q+5 q^{2}+\cdots,
$$

leading to the same result as in [1]

$$
\sigma_{\mathrm{MHS}_{2}, 5}(q)=4 q^{-1}+7+4 q
$$

with the same expression also for $\sigma_{\mathrm{CHS}_{2}, 4}(q)$.

\section{Higher derivative conformal scalar fields}

Here we will illustrate the relations (3.1),(3.2),(3.5) on the example of Weyl-covariant scalar theory with kinetic operator $\widehat{\Delta}_{(2 r)}=-\left(\nabla^{2}\right)^{r}+\ldots$ where dots stand for curvature dependent terms.

The GJMS operators $\widehat{\Delta}_{(2 r)}$ naturally exist in a technical sense for $r \leq d / 2$ (see [51] and refs. there).This means that their definition in generic dimension $d$ involves terms 
whose coefficients have poles in $d$ when $r>d / 2$. For instance, for $r=3$, there are tensor structures with coefficient $\frac{1}{d-4}$ forbidding a naive extension to $4 \mathrm{~d}$ case. These obstructions are proportional to Bach tensor and vanish for the Einstein spaces with $R_{m n}=\frac{R}{d} g_{m n}$. In this case it is possible to construct generalized Gover-GJMS operators (defined beyond critical order) but the resulting expressions are non-natural in technical sense [52]. For all orders (below, at, and beyond critical order) the Gover-GJMS operators factorise as in (C.10) below. In the conformally flat spaces (that need not be Einstein in general), there is no obstruction in going beyond the critical order $r=d / 2$.

\section{C.1 Partition function on $S^{1} \times S^{3}$}

Let us first consider the general case of the space $S^{q} \times S^{p}$ is conformally flat if defined with indefinite $(p, q)$ signature metric (here the spheres have unit radius and $d=p+q$ ). Then one can show that for $r=2 N$ [53]

$$
\begin{aligned}
& \widehat{\Delta}_{(4 N)}=\prod_{k=1}^{N}\left[\left(\mathcal{O}_{p}-\mathcal{O}_{q}\right)^{2}-2(2 k-1)^{2}\left(\mathcal{O}_{p}+\mathcal{O}_{q}\right)+(2 k-1)^{4}\right] \\
& \widehat{\Delta}_{(4 N+2)}=\left(\mathcal{O}_{p}-\mathcal{O}_{q}\right) \prod_{k=1}^{N}\left[\left(\mathcal{O}_{p}-\mathcal{O}_{q}\right)^{2}-2(2 k)^{2}\left(\mathcal{O}_{p}+\mathcal{O}_{q}\right)+(2 k)^{4}\right]
\end{aligned}
$$

where $\mathcal{O}_{p} \equiv-\nabla_{S^{p}}^{2}+\frac{1}{4}(p-1)^{2}$. For example, in the special case of $\Delta_{(4)}$ in $d=4$ we have for a general curved background $[49,54]$

$$
\widehat{\Delta}_{(4)}=-\left(\nabla^{2}\right)^{2}+2\left(R^{m n}-\frac{1}{3} g^{m n} R\right) \nabla_{m} \nabla_{n} .
$$

Then for $S^{2} \times S^{\prime 2}$ with $(++--)$ signature we get ${ }^{24}$

$$
\widehat{\Delta}_{(4)}=\left(-\nabla_{S^{2}}^{2}+\nabla_{S^{\prime 2}}^{2}\right)^{2}+2\left(\nabla_{S^{2}}^{2}+\nabla_{S^{\prime 2}}^{2}\right)=\left(\mathcal{O}_{2}-\mathcal{O}_{2}^{\prime}\right)^{2}-2\left(\mathcal{O}_{2}+\mathcal{O}_{2}^{\prime}\right)+1,
$$

in agreement with (C.1) where $\mathcal{O}_{2}=-\nabla_{S^{2}}^{2}+\frac{1}{4}$.

For conformally flat but not Einstein space $S^{1} \times S^{3}$ with $(-+++)$ signature we have for the spectra of $\mathcal{O}_{p}$ in (C.1)

$$
\mathcal{O}_{1} \rightarrow w, \quad \mathcal{O}_{3} \rightarrow n(n+2)+1=(n+1)^{2}, n=0,1,2, \ldots
$$

For $r=2 N$ the factorisation of $\Delta_{(4 N)}$ in (C.1) leads to the energy eigenvalues $w$ represented by

$$
w_{n}=n+2-r+2 k, \quad k=0, \ldots, r-1,
$$

so that the final one-particle partition function for a GJMS scalar field is

$$
\mathcal{Z}_{\mathrm{GJMS}_{r}}\left(S^{1} \times S^{3} ; q\right)=\sum_{n=0}^{\infty} \sum_{k=0}^{r-1}(n+1)^{2} q^{n+2-r+2 k}=\frac{q^{2-r}-q^{2+r}}{(1-q)^{4}} .
$$

\footnotetext{
${ }^{24}$ Here $R_{m n}= \pm g_{m n}=g_{m n}^{(0)}$, where $g_{m n}^{(0)}$ is the metric of a standard 2-sphere and the sign depends on whether $(m n)$ are in the first or second sphere. Thus $\nabla^{2}=-\nabla_{S^{2}}^{2}+\nabla_{S^{2}}^{2}$ and $R^{m n} \nabla_{m} \nabla_{n}=\nabla_{S^{2}}^{2}+\nabla_{S^{2}}^{2}$.
} 
This is the same as the partition function that counts descendants of a conformal scalar operator in flat space modulo its equation of motion. In general dimension $d$, for a GJMS scalar $\phi$ with canonical dimension $\frac{1}{2}(d-2 r)$ and equations of motion $\partial^{2 r} \phi=0$ of complementary dimension $\frac{1}{2}(d+2 r)$ we get

$$
\mathcal{Z}_{\mathrm{GJMS}_{r}}\left(S^{1} \times S^{d-1} ; q\right)=\frac{q^{\frac{d}{2}-r}-q^{\frac{d}{2}+r}}{(1-q)^{d}} .
$$

\section{C.2 Partition function on $\mathrm{AdS}_{d}$}

The discussion in section 3.1 may be generalized by considering a massive scalar field in AdS $_{d+1}$ with $\Delta_{d+1}^{ \pm}=\frac{1}{2}(d \pm 2 r)$ (with $r=2,3, \ldots$ ) for which the associated $d$-dimensional boundary conformal field is the higher derivative conformal scalar with canonical dimension $\Delta_{d+1}^{-}=\frac{1}{2}(d-2 r)$ and the kinetic operator $\widehat{\Delta}_{(2 r)}$. Here (3.7) is replaced by

$$
\mathcal{Z}_{0, r}^{ \pm}\left(\operatorname{AdS}_{d+1} ; q\right)=\frac{q^{\frac{1}{2}(d \pm 2 r)}}{(1-q)^{d}} .
$$

On a generic $d$-dimensional Einstein space $\widehat{\Delta}_{(2 r)}$ factorizes as follows [52]

$$
\widehat{\Delta}_{(2 r)}=\prod_{k=1}^{r}\left(-\nabla^{2}+\frac{\left(\frac{d}{2}-k\right)\left(\frac{d}{2}+k-1\right)}{d(d-1)} R\right) .
$$

For $\mathrm{AdS}_{d}$ case we have $R=-d(d-1)$ and using (2.2) then find that for each massive Laplacian factor in (C.10) the associated dimensions are

$$
\Delta_{d, k}^{+}=\frac{d+2 k}{2}-1, \quad \Delta_{d, k}^{-}=\frac{d-2 k}{2}, \quad k=1, \ldots, r .
$$

Hence, (3.10) is generalized to (there is no gauge invariance so that $\sigma_{d}=0$ and $\widetilde{\mathcal{Z}}^{-}=\mathcal{Z}^{-}$)

$$
\begin{aligned}
& \mathcal{Z}_{\mathrm{GJMS}_{r}}^{+}\left(\operatorname{AdS}_{d} ; q\right)=\sum_{k=1}^{r} \frac{q^{\Delta_{d, k}^{+}}}{(1-q)^{d-1}}=\frac{q^{d / 2}\left(1-q^{r}\right)}{(1-q)^{d}} \\
& \mathcal{Z}_{\mathrm{GJMS}_{r}}^{-}\left(\operatorname{AdS}_{d} ; q\right)=(-1)^{d+1} \mathcal{Z}_{\mathrm{GJMS}_{r}}^{+}\left(\operatorname{AdS}_{d} ; q^{-1}\right) .
\end{aligned}
$$

Comparing (C.9) to (C.12),(C.13) one checks again the relation (3.1),(3.5), (3.1)

$$
\mathcal{Z}_{0, r}^{-}\left(\mathrm{AdS}_{d+1} ; q\right)-\mathcal{Z}_{0, r}^{+}\left(\operatorname{AdS}_{d+1} ; q\right)=\mathcal{Z}_{\mathrm{GJMS}_{r}}^{-}\left(\mathrm{AdS}_{d} ; q\right)+\mathcal{Z}_{\mathrm{GJMS}_{r}}^{+}\left(\mathrm{AdS}_{d} ; q\right) .
$$

We can also demonstrate the relation (3.2) by using (C.8) and (C.12),(C.13) to check that

$$
\mathcal{Z}_{\mathrm{GJMS}_{r}}\left(S^{1} \times S^{d-1} ; q\right)=\mathcal{Z}_{\mathrm{GJMS}_{r}}^{-}\left(\mathrm{AdS}_{d} ; q\right)+\mathcal{Z}_{\mathrm{GJMS}_{r}}^{+}\left(\operatorname{AdS}_{d} ; q\right) .
$$

\section{Fermionic conformal higher spin fields}

The discussion of partition function relations for the bosonic CHS fields may be extended to the $4 \mathrm{~d}$ fermionic ones (see [20]). These are boundary conterparts for the massless spin $s$ fermionic higher spin fields in $\mathrm{AdS}_{5}$ to the $\mathrm{SO}(2,4)$ representation

$$
\mathrm{MHS}_{\mathrm{s}}=\left(\mathrm{s}+\frac{5}{2} ; \frac{\mathrm{s}}{2}, \frac{\mathrm{s}+1}{2}\right)+\left(\mathrm{s}+\frac{5}{2} ; \frac{\mathrm{s}+1}{2}, \frac{\mathrm{s}}{2}\right), \quad \mathrm{s} \equiv \mathrm{s}-\frac{1}{2}=0,1,2, \ldots
$$


Here $\mathrm{s}=0$ corresponds to spin $\frac{1}{2}$ fermion, $\mathrm{s}=1$ to conformal gravitino, etc. Recalling that for the massless $S O(2,4)$ representation $\left(2+j_{1}+j_{2} ; j_{1}, j_{2}\right)$ we have ${ }^{25}$

$$
\mathcal{Z}_{\left(2+j_{1}+j_{2} ; j_{1}, j_{2}\right)}^{+}\left(\mathrm{AdS}_{5} ; q\right)=\frac{q^{2+j_{1}+j_{2}}}{(1-q)^{4}}\left[\left(2 j_{1}+1\right)\left(2 j_{2}+1\right)-4 q j_{1} j_{2}\right]
$$

we obtain (see Eq. (2.17) in [3])

$$
\mathcal{Z}_{\mathrm{MHS}_{\mathrm{s}}}^{+}\left(\mathrm{AdS}_{5} ; q\right)=\frac{2(\mathrm{~s}+1)(\mathrm{s}+2) q^{\frac{5}{2}+\mathrm{s}}-2 \mathrm{~s}(\mathrm{~s}+1) q^{\frac{7}{2}+\mathrm{s}}}{(1-q)^{4}} .
$$

Applying the "reconstruction" algorithm in Appendix (A) or using the explicit factorized form of the fermionic CHS partition function on $S^{4}$ [20] and thus also on $\mathrm{AdS}_{4}$ one may find that

$$
\mathcal{Z}_{\mathrm{CSH}_{\mathrm{s}}}^{+}\left(\mathrm{AdS}_{4} ; q\right)=2 \frac{(\mathrm{s}+1)\left(q^{\frac{3}{2}}+q^{\frac{5}{2}}\right)-(\mathrm{s}+1)(\mathrm{s}+2) q^{\frac{5}{2}+\mathrm{s}}+\mathrm{s}(\mathrm{s}+1) q^{\frac{7}{2}+\mathrm{s}}}{(1-q)^{4}} .
$$

One then concludes that the relation (3.5) is again satisfied

$$
\widetilde{\mathcal{Z}}_{\mathrm{MHS}_{\mathrm{s}}}^{-}\left(\mathrm{AdS}_{5} ; q\right)-\mathcal{Z}_{\mathrm{MHS}_{\mathrm{s}}}^{+}\left(\mathrm{AdS}_{5} ; q\right)=\widetilde{\mathcal{Z}}_{\mathrm{CHS}_{\mathrm{s}}}^{-}\left(\mathrm{AdS}_{4} ; q\right)+\mathcal{Z}_{\mathrm{CHS}_{\mathrm{s}}}^{+}\left(\mathrm{AdS}_{4} ; q\right) .
$$

In addition, the partition function $\mathcal{Z}_{\mathrm{CHS}_{\mathrm{s}}}\left(S^{1} \times S^{3} ; q\right)$ was found in [3] (see Eq. (2.26) there). Comparing it with (D.4), we conclude that the relation (4.14) holds also for the fermionic $\mathrm{CSH}_{\mathrm{s}}$ family, i.e.

$$
\mathcal{Z}_{\mathrm{CHS}_{\mathrm{s}}}\left(S^{1} \times S^{3} ; q\right)=2 \mathcal{Z}_{\mathrm{CHS}_{\mathrm{s}}}^{+}\left(\mathrm{AdS}_{4} ; q\right)
$$

\section{E Conformal antisymmetric tensor fields in $4 \mathrm{~d}$}

The Weyl-covariant Lagrangian for the conformal antisymmetric tensor field $T_{\mu \nu}$ on a generic curved $4 \mathrm{~d}$ background is [29]

$$
\mathscr{L}=\left(\nabla^{\mu} T_{\mu \nu}\right)^{2}-\frac{1}{4}\left(\nabla_{\mu} T_{\rho \sigma}\right)^{2}-R_{\mu \nu} T^{\mu \lambda} T_{\lambda}^{v}+\frac{1}{8} R T_{\mu \nu}^{2}+\frac{1}{2} R_{\mu \alpha \nu \beta} T^{\mu v} T^{\alpha \beta} .
$$

This conformal field in flat $4 \mathrm{~d}$ space corresponds in $\mathrm{AdS}_{5}$ to a massive spin 1 theory with representation content $\mathrm{HS}=(3 ; 1,0) \oplus(3 ; 0,1)$ and no gauge invariance. The Lagrangian (E.1) restricted to $\mathrm{AdS}_{4}$ background gives the kinetic operator that factorizes into vector operators as discussed in [48]. The thermal partition function on $S^{1} \times S^{3}$ may be found in eq. (B.26) of [1]. As a result,

$$
\mathcal{Z}_{\mathrm{HS}}^{+}\left(\mathrm{AdS}_{5} ; q\right)=\frac{6 q^{3}}{(1-q)^{4}}, \quad \mathcal{Z}_{\mathrm{T}}^{+}\left(\mathrm{AdS}_{4} ; q\right)=\frac{6 q^{2}}{(1-q)^{3}}, \quad \mathcal{Z}_{\mathrm{T}}\left(S^{1} \times S^{3} ; q\right)=\frac{6 q-6 q^{3}}{(1-q)^{4}} .
$$

One finds then that (3.5) is satisfied

$$
\widetilde{\mathcal{Z}}_{\mathrm{HS}}^{-}\left(\mathrm{AdS}_{5} ; q\right)-\mathcal{Z}_{\mathrm{HS}}^{+}\left(\mathrm{AdS}_{5} ; q\right)=\widetilde{\mathcal{Z}}_{\mathrm{T}}^{-}\left(\mathrm{AdS}_{4} ; q\right)+\mathcal{Z}_{\mathrm{T}}^{+}\left(\mathrm{AdS}_{4} ; q\right)
$$

\footnotetext{
${ }^{25}$ The gauge subtraction in (D.2) is present only for $\mathrm{s}>0$ but since it happens to vanish for $\mathrm{s}=0$ this formula is general.
} 
but there is no analogue of (4.14).

Let us elaborate on the derivation of $\mathcal{Z}_{\mathrm{T}}^{+}\left(\mathrm{AdS}_{4} ; q\right)$ in (E.2). The antisymmetric tensor partition function on $S^{4}$ is [48]

$$
Z_{\mathrm{T}}\left(S^{4}\right)=\left[\operatorname{det} \widehat{\Delta}_{(1,0)}(4) \operatorname{det} \widehat{\Delta}_{(0,1)}(4)\right]^{-1 / 2},
$$

where the 2nd order operator $\widehat{\Delta}_{\left(j_{1}, j_{2}\right)}\left(M^{2}\right)$ (cf. (2.1)) acts on a field in an irreducible $S O(1,3)$ representation $\left(j_{1}, j_{2}\right)$ (see, e.g., $\left.[55,20]\right)$. Similar partition function is found on $\mathrm{AdS}_{4}$ where the mass term is related the corresponding conformal dimension $\Delta_{4}^{ \pm}$by the following generalization of $(2.2)^{26}$

$$
\Delta_{4}^{ \pm}\left(\Delta_{4}^{ \pm}-3\right)-j_{1}\left(j_{1}+1\right)-j_{2}\left(j_{2}+1\right)=-M^{2} .
$$

For $M^{2}=4$ and $\left(j_{1}, j_{2}\right)=(0,1)$ or $(1,0)$ as in (E.4) this gives $\Delta_{4}^{+}=2$ and $\Delta_{4}^{-}=1$. Therefore, $\mathcal{Z}_{\mathrm{T}}^{+}\left(\mathrm{AdS}_{4} ; q\right)=\frac{6 q^{2}}{(1-q)^{3}}$, in agreement with (E.2) (the factor 6 is the dimension of $(1,0) \oplus(0,1)$ representation).

Note that the partition function in (E.4) leads to the correct value of the conformal a-anomaly coefficient for $T_{\mu \nu}$. Using Eqs. (3.34)-(3.35) of [20], the contribution to the aanomaly from $\widehat{\Delta}_{\left(j_{1}, j_{2}\right)}\left(M^{2}\right)$ is found to be

$$
\mathrm{a}_{\left(j_{1}, j_{2}\right)}\left(M^{2}\right)=\frac{1}{720}\left(2 j_{1}+1\right)\left(2 j_{2}+1\right)\left[10\left(j_{1}\left(j_{1}+1\right)+j_{2}\left(j_{2}+1\right)\right)-15 M^{4}+60 M^{2}-58\right],
$$

so that $\mathrm{a}(T)=\widehat{\mathrm{a}}_{(1,0)}(4)+\widehat{\mathrm{a}}_{(0,1)}(4)=-\frac{19}{60}$, in agreement with $[48,3] \cdot{ }^{27}$ Indeed, the general form of (4.6) is [1]

$$
\begin{aligned}
& \mathrm{a}\left(\Delta_{5} ; j_{1}, j_{2}\right)=\frac{1}{720}\left(2 j_{1}+1\right)\left(2 j_{2}+1\right)\left(\Delta_{5}-2\right) \\
& \times\left[-3\left(\Delta_{5}-2\right)^{4}+10\left(j_{1}^{2}+j_{2}^{2}+j_{1}+j_{2}+\frac{1}{2}\right)\left(\Delta_{5}-2\right)^{2}-15\left(j_{1}-j_{2}\right)^{2}\left(j_{1}+j_{2}+1\right)^{2}\right],
\end{aligned}
$$

and we again get $\mathrm{a}(3 ; 1,0)+\mathrm{a}(3 ; 0,1)=-\frac{19}{60}$.

One can repeat the above discussion for a conformal $4 \mathrm{~d}$ field $T_{p}$ transforming in the $(p, 0) \oplus(0, p)$ representation of the $S O(1,3){ }^{28}$ We may start in $\mathrm{AdS}_{5}$ with a $5 \mathrm{~d}$ field in $\left(\Delta_{5} ; p, 0\right) \oplus\left(\Delta_{5} ; 0, p\right)$ representation (to be denoted as $\left.\mathrm{HS}_{p}\right)$. It should correspond to a conformal field in $\mathrm{AdS}_{4}$ with the canonical dimension $4-\Delta_{5}$ and thus with the kinetic term $T_{p} \square^{\Delta_{5}-2} T_{p}+\ldots$. The correspondence rule (4.1) here reads as

$$
\left(\Delta_{5} ; p, 0\right) \oplus\left(\Delta_{5} ; 0, p\right) \rightarrow \mathrm{Z}_{\mathrm{T}_{p}}\left(\mathrm{AdS}_{4}\right)=\prod_{k=1}^{\Delta_{5}-2}\left[\operatorname{det} \widehat{\Delta}_{(p, 0) \oplus(0, p)}(2+p(p+1)-k(k-1))\right]^{-1 / 2}
$$

\footnotetext{
${ }^{26}$ The explicit dependence on $j_{1,2}$ through the Casimir of $S O(4)$ comes from the particular definition of the operator, see Eq. (3.5) of [20].

${ }^{27}$ As explained in [56], it is also possible to express $T_{\mu v}$ in terms of two spin 1 vector fields and thus write the partition function in the form (see Eq.(5) of [48], cf. (4.25)) $Z_{\mathrm{T}}\left(S^{4}\right)=C\left[\operatorname{det} \widehat{\Delta}_{1 \perp}(3)\right]^{-1}$, where $C$ accounts for the zero mode contributions (cf. discussion after Eq.(6.18) in [29]). This zero mode factor is essential to reproduce the correct value for the a-anomaly giving extra $-\frac{1}{2}$ shift: using (4.7) to find $\widehat{a}_{1 \perp}(3)$ we get $\widehat{\mathrm{a}}(T)=2 \widehat{\mathrm{a}}_{1 \perp}(3)-\frac{1}{2}=-\frac{19}{60}$.

${ }^{28}$ Fields transforming in the $(p, 0) \oplus(0, p)$ representation are Weyl-like tensors (see, e.g., [57]). They may be represented as rank $2 p$ tensors $T_{\mu_{1} v_{1} \mu_{2} v_{2} \cdots \mu_{p} v_{p}}$ antisymmetric in each pair $\mu_{i} v_{i}$, totally symmetric with respect to the exchange of the pairs $\left(\mu_{i} v_{i}\right)$ and $\left(\mu_{j} v_{j}\right)$, traceless, and obeying a generalized "Bianchi identity" $T_{\ldots[\mu \nu \rho]}=0$.
} 
Using (E.6) and (E.7) one finds that the equality of the a-anomaly coefficients implied by (E.8) indeed holds

$$
\mathrm{a}\left(\Delta_{5} ; p, 0\right)+\mathrm{a}\left(\Delta_{5} ; 0, p\right)=2 \sum_{k=1}^{\Delta_{5}-1} \mathrm{a}_{(p, 0)}(2+p(p+1)-k(k-1)) .
$$

From (E.5) we find that the dimension corresponding according to (2.2) to the $k$-th operator in the r.h.s. of (E.8) is $\Delta_{4}^{(k)}=k+1$, so that from (2.3) we get

$$
\begin{aligned}
\mathcal{Z}_{\mathrm{HS}_{p}}^{+}\left(\mathrm{AdS}_{5} ; q\right) & =\frac{2(2 p+1) q^{\Delta_{5}}}{(1-q)^{4}} \\
\mathcal{Z}_{\mathrm{T}_{p}}^{+}\left(\mathrm{AdS}_{4} ; q\right) & =\frac{2(2 p+1)}{(1-q)^{3}} \sum_{k=1}^{\Delta_{5}-2} q^{k+1}=\frac{2(2 p+1)}{(1-q)^{4}}\left(q^{2}-q^{\Delta_{5}}\right) .
\end{aligned}
$$

Thus once again we get the relation (3.5)

$$
\widetilde{\mathcal{Z}}_{\mathrm{HS}_{p}}^{-}\left(\mathrm{AdS}_{5} ; q\right)-\mathcal{Z}_{\mathrm{HS}_{p}}^{+}\left(\mathrm{AdS}_{5} ; q\right)=\widetilde{\mathcal{Z}}_{\mathrm{T}_{p}}^{-}\left(\mathrm{AdS}_{4} ; q\right)+\mathcal{Z}_{\mathrm{T}_{p}}^{+}\left(\mathrm{AdS}_{4} ; q\right)
$$

\section{References}

[1] M. Beccaria, X. Bekaert, and A. A. Tseytlin, Partition function of free conformal higher spin theory, JHEP 1408 (2014) 113, [arXiv: 1406 . 3542].

[2] S. Giombi, I. R. Klebanov, S. S. Pufu, B. R. Safdi, and G. Tarnopolsky, AdS Description of Induced Higher-Spin Gauge Theory, JHEP 1310 (2013) 016, [arXiv: 1306 . 5242].

[3] M. Beccaria and A. A. Tseytlin, Higher spins in $A d S_{5}$ at one loop: vacuum energy, boundary conformal anomalies and AdS/CFT, JHEP 1411 (2014) 114, [arXiv: 1410 . 3273].

[4] P. Breitenlohner and D. Z. Freedman, Positive Energy in anti-De Sitter Backgrounds and Gauged Extended Supergravity, Phys. Lett. B115 (1982) 197.

[5] B. E. W. Nilsson, Aspects of topologically gauged M2-branes with six supersymmetries: towards a 'sequential AdS/CFT'?, in Quantum theory and symmetries. Proceedings, 7th International Conference, QTS7, Prague, Czech Republic, August 7-13, 2011, 2012. arXiv:1203. 5090.

[6] T. Ohl and C. F. Uhlemann, Saturating the unitarity bound in AdS/CFT (AdS), JHEP 05 (2012) 161, [arXiv: 1204.2054].

[7] A. Karch and L. Randall, Locally localized gravity, JHEP 05 (2001) 008, [hep-th/0011156].

[8] G. Compere and D. Marolf, Setting the boundary free in AdS/CFT, Class. Quant. Grav. 25 (2008) 195014, [arXiv:0805.1902].

[9] O. Aharony, D. Marolf, and M. Rangamani, Conformal field theories in anti-de Sitter space, JHEP 02 (2011) 041, [arXiv: 1011.6144].

[10] T. Andrade and C. F. Uhlemann, Beyond the unitarity bound in AdS/CFT (A)dS, JHEP 01 (2012) 123, [arXiv: 1111.2553].

[11] K. Hinterbichler, J. Stokes, and M. Trodden, Holographic CFTs on maximally symmetric spaces: correlators, integral transforms and applications, Phys. Rev. D92 (2015), no. 6 065025, [arXiv: 1505.05513]. 
[12] R. R. Metsaev, Massive fields in AdS(3) and compactification in AdS space time, Nucl. Phys. Proc. Suppl. 102 (2001) 100-106, [hep-th/0103088]. [,100(2000)].

[13] A. Yu. Artsukevich and M. A. Vasiliev, On Dimensional Degression in AdS(d), Phys. Rev. D79 (2009) 045007, [arXiv : 0810.2065].

[14] J. S. Dowker, Arbitrary Spin Theory in the Einstein Universe, Phys. Rev. D28 (1983) 3013.

[15] S. W. Hawking and H. S. Reall, Charged and rotating AdS black holes and their CFT duals, Phys. Rev. D61 (2000) 024014, [hep-th/9908109].

[16] R. R. Metsaev, Ordinary-derivative formulation of conformal totally symmetric arbitrary spin bosonic fields, JHEP 1206 (2012) 062, [arXiv : 0709 . 4392].

[17] R. R. Metsaev, Lowest eigenvalues of the energy operator for totally (anti)symmetric massless fields of the n-dimensional anti-de Sitter group, Class.Quant.Grav. 11 (1994) L141-L145.

[18] R. R. Metsaev, Massless mixed symmetry bosonic free fields in d-dimensional anti-de Sitter space-time, Phys.Lett. B354 (1995) 78-84.

[19] R. R. Metsaev, Massive totally symmetric fields in AdS ${ }_{d}$, Phys.Lett. B590 (2004) 95-104, [hep-th/0312297].

[20] A. A. Tseytlin, On partition function and Weyl anomaly of conformal higher spin fields, Nucl.Phys. B877 (2013) 598-631, [arXiv: 1309.0785].

[21] A. A. Tseytlin, Weyl anomaly of conformal higher spins on six-sphere, Nucl.Phys. B877 (2013) 632-646, [arXiv:1310.1795].

[22] M. Irakleidou and I. Lovrekovic, Conformal gravity 1-loop partition function, arXiv:1512.07130

[23] I. Lovrekovic, One loop partition function of six dimensional conformal gravity using heat kernel on AdS, arXiv: 1512.00858.

[24] R. Gopakumar, R. K. Gupta, and S. Lal, The Heat Kernel on AdS, JHEP 1111 (2011) 010, [arXiv:1103.3627].

[25] R. K. Gupta and S. Lal, Partition Functions for Higher-Spin theories in AdS, JHEP 1207 (2012) 071, [arXiv: 1205.1130].

[26] S. Giombi, I. R. Klebanov, and A. A. Tseytlin, Partition Functions and Casimir Energies in Higher Spin $A d S_{d+1} / C F T_{d}$, Phys. Rev. D90 (2014), no. 2 024048, [arXiv: 1402 . 5396].

[27] J. Erdmenger and H. Osborn, Conformally covariant differential operators: Symmetric tensor fields, Class.Quant.Grav. 15 (1998) 273-280, [gr-qc/9708040].

[28] M. Beccaria and A. Tseytlin, On higher spin partition functions, J.Phys. A48 (2015), no. 27 275401, [arXiv: 1503.08143].

[29] E. S. Fradkin and A. A. Tseytlin, Conformal supergravity, Phys.Rept. 119 (1985) 233-362.

[30] M. A. Vasiliev, Bosonic conformal higher-spin fields of any symmetry, Nucl. Phys. B829 (2010) 176-224, [arXiv:0909.5226].

[31] X. Bekaert and M. Grigoriev, Higher order singletons, partially massless fields and their boundary values in the ambient approach, Nucl.Phys. B876 (2013) 667-714, [arXiv: 1305. 0162].

[32] G. Barnich, X. Bekaert, and M. Grigoriev, Notes on conformal invariance of gauge fields, J. Phys. A48 (2015), no. 50 505402, [arXiv: 1506. 00595]. 
[33] M. Beccaria and A. A. Tseytlin, Conformal a-anomaly of some non-unitary $6 d$ superconformal theories, JHEP 09 (2015) 017, [arXiv: 1506 . 08727].

[34] F. Dolan, Character formulae and partition functions in higher dimensional conformal field theory, J.Math.Phys. 47 (2006) 062303, [hep-th/0508031].

[35] R. R. Metsaev, All conformal invariant representations of d-dimensional anti-de Sitter group, Mod. Phys. Lett. A10 (1995) 1719-1731.

[36] R. Metsaev, Conformal self-dual fields, J.Phys. A43 (2010) 115401, [arXiv: 0812 . 2861].

[37] A. A. Tseytlin, On limits of superstring in $A d S_{5} \times S^{5}$, Theor.Math.Phys. 133 (2002) 1376-1389, [hep-th/0201112].

[38] H. Osborn and A. C. Petkou, Implications of conformal invariance in field theories for general dimensions, Annals Phys. 231 (1994) 311-362, [hep-th/9307010].

[39] R. G. Leigh and A. C. Petkou, SL(2,Z) action on three-dimensional CFTs and holography, JHEP 12 (2003) 020, [hep-th/0309177].

[40] C. N. Pope and P. K. Townsend, Conformal Higher Spin in (2+1)-dimensions, Phys. Lett. B225 (1989) 245.

[41] E. S. Fradkin and V. Ya. Linetsky, A Superconformal Theory of Massless Higher Spin Fields in D $=(2+1)$, Mod. Phys. Lett. A4 (1989) 731. [Annals Phys.198,293(1990)].

[42] B. E. W. Nilsson, On the conformal higher spin unfolding equation for a three-dimensional self-interacting scalar field, arXiv:1506.03328.

[43] S. Deser, R. Jackiw, and S. Templeton, Topologically Massive Gauge Theories, Annals Phys. 140 (1982) 372-411. [Annals Phys.281,409(2000)].

[44] J. H. Horne and E. Witten, Conformal Gravity in Three-dimensions as a Gauge Theory, Phys. Rev. Lett. 62 (1989) 501-504.

[45] H. Afshar, B. Cvetkovic, S. Ertl, D. Grumiller, and N. Johansson, Conformal Chern-Simons holography - lock, stock and barrel, Phys. Rev. D85 (2012) 064033, [arXiv: 1110 . 5644].

[46] E. Witten, SL(2,Z) action on three-dimensional conformal field theories with Abelian symmetry, hep-th/0307041.

[47] A. A. Tseytlin, Effective action in de Sitter space and conformal supergravity, Yad.Fiz. 39 (1984), no. 6 1606-1615.

[48] E. S. Fradkin and A. A. Tseytlin, Instanton zero modes and beta functions in supergravities. 2. Conformal supergravity, Phys.Lett. B134 (1984) 307.

[49] E. S. Fradkin and A. A. Tseytlin, One Loop Beta Function in Conformal Supergravities, Nucl.Phys. B203 (1982) 157.

[50] S. Deser and R. I. Nepomechie, Gauge Invariance Versus Masslessness in De Sitter Space, Annals Phys. 154 (1984) 396.

[51] A. Juhl, Explicit formulas for GJMS-operators and $\$ Q \$$-curvatures, ArXiv e-prints (Aug., 2011) [arXiv: 1108.0273].

[52] A. R. Gover, Laplacian operators and Q-curvature on conformally Einstein manifolds, math/0506037.

[53] A. Juhl, On conformally covariant powers of the Laplacian, ArXiv e-prints (May, 2009) [arXiv: 0905.3992]. 
[54] S. Paneitz, A Quartic Conformally Covariant Differential Operator for Arbitrary Pseudo-Riemannian Manifolds (Summary), arXiv:0803.4331.

[55] S. Christensen and M. Duff, New Gravitational Index Theorems and Supertheorems, Nucl.Phys. B154 (1979) 301.

[56] E. S. Fradkin and A. A. Tseytlin, Asymptotic freedom in extended conformal supergravities, Phys. Lett. B110 (1982) 117-122.

[57] S. Gómez-Ávila and M. Napsuciale, Covariant basis induced by parity for the $(j, 0) \oplus(0, j)$ representation, Phys. Rev. D88 (2013), no. 9 096012, [arXiv: 1307.4711]. 